\title{
Geometrically constructed bases for homology of partition lattices of types $A, B$ and $D$
}

\author{
Anders Björner* \\ Royal Institute of Technology, Department of Mathematics \\ S-100 44 Stockholm, Sweden \\ bjorner@math.kth.se \\ Michelle L. Wachs ${ }^{\dagger}$ \\ University of Miami, Department of Mathematics \\ Coral Gables, FL 33124, USA \\ wachs@math.miami.edu
}

Submitted: Jan 1, 2004; Accepted: Apr 17, 2004; Published: Jun 3, 2004

MR Subject Classifications: 05E25, 52C35, 52C40

Dedicated to Richard Stanley on the occasion of his 60th birthday

\begin{abstract}
We use the theory of hyperplane arrangements to construct natural bases for the homology of partition lattices of types $A, B$ and $D$. This extends and explains the "splitting basis" for the homology of the partition lattice given in [20], thus answering a question asked by R. Stanley.

More explicitly, the following general technique is presented and utilized. Let $\mathcal{A}$ be a central and essential hyperplane arrangement in $\mathbb{R}^{d}$. Let $R_{1}, \ldots, R_{k}$ be the bounded regions of a generic hyperplane section of $\mathcal{A}$. We show that there are induced polytopal cycles $\rho_{R_{i}}$ in the homology of the proper part $\bar{L}_{\mathcal{A}}$ of the intersection lattice such that $\left\{\rho_{R_{i}}\right\}_{i=1, \ldots, k}$ is a basis for $\widetilde{H}_{d-2}\left(\bar{L}_{\mathcal{A}}\right)$. This geometric method for constructing combinatorial homology bases is applied to the Coxeter arrangements of types $A, B$ and $D$, and to some interpolating arrangements.
\end{abstract}

\section{Introduction}

In [20] Wachs constructs a basis for the homology of the partition lattice $\Pi_{n}$ via a certain natural "splitting" procedure for permutations. This basis has very favorable properties

${ }^{*}$ Supported in part by Göran Gustafsson Foundation for Research in Natural Sciences and Medicine.

${ }^{\dagger}$ Supported in part by National Science Foundation grants DMS-9701407 and DMS-0073760. 
with respect to the representation of the symmetric group $S_{n}$ on $\widetilde{H}_{n-3}\left(\Pi_{n}, \mathbb{C}\right)$, a representation that had earlier been studied by Stanley [19], Hanlon [14] and many others. It also is the shelling basis for a certain EL-shelling of the partition lattice given in [20, Section 6]. This basis has connections to the free Lie algebra as well; see [21].

We now give a brief description of the splitting basis of [20]. For each $\omega \in S_{n}$, let $\Pi_{\omega}$ be the subposet of $\Pi_{n}$ consisting of partitions obtained by splitting $\omega$. In Figure 1 the subposet $\Pi_{3124}$ of $\Pi_{4}$ is shown. Each poset $\Pi_{\omega}$ is isomorphic to the face lattice of an $(n-2)$-dimensional simplex. Therefore $\Delta\left(\bar{\Pi}_{\omega}\right)$, the order complex of the proper part of $\Pi_{\omega}$, is an $(n-3)$-sphere embedded in $\Delta\left(\bar{\Pi}_{n}\right)$, and hence it determines a fundamental cycle $\rho_{\omega} \in \tilde{H}_{n-3}\left(\bar{\Pi}_{n}\right)$. In [20] it is shown that a certain subset of $\left\{\rho_{\omega} \mid \omega \in S_{n}\right\}$ forms a basis for $\tilde{H}_{n-3}\left(\bar{\Pi}_{n}\right)$; namely, the set of all $\rho_{\omega}$ such that $\omega$ fixes $n$.

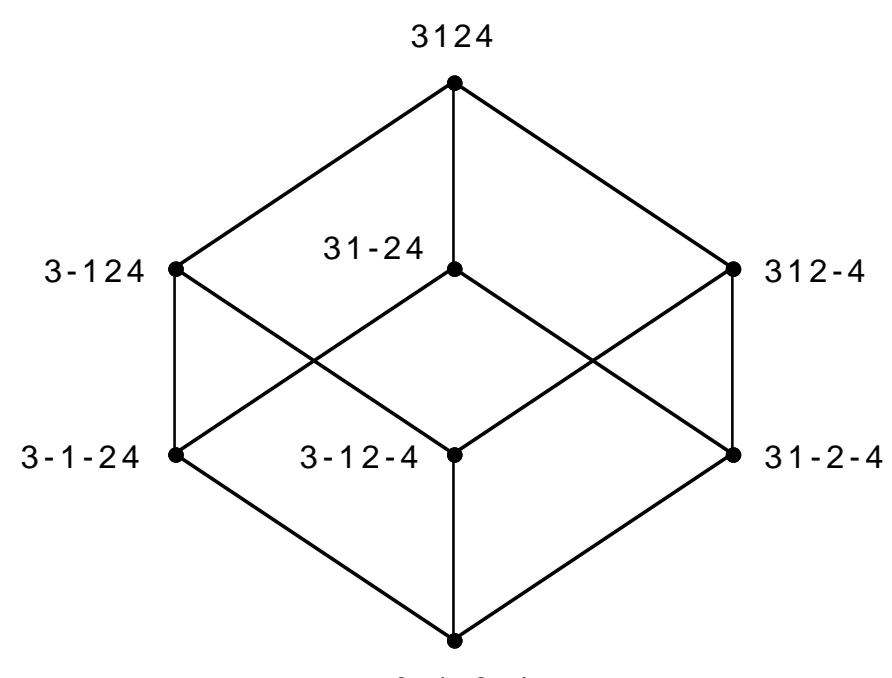

$3-1-2-4$

Figure 1

The partition lattice is the intersection lattice of the type $A$ Coxeter arrangement. The original motivation for this paper was to explain and generalize to other Coxeter groups, the splitting basis for $\Pi_{n}$. Taking a geometric point of view we give such an explanation, which then leads to the construction of "splitting bases" also for the intersection lattices of Coxeter arrangements of types $B$ and $D$ and of some interpolating arrangements. Our technique is general in that it gives a way to construct a basis for the homology of the intersection lattice of any real hyperplane arrangement.

The intersection lattice of the type $B$ Coxeter arrangement is isomorphic to the signed partition lattice $\Pi_{n}^{B}$. Its elements are signed partitions of $\{0,1, \ldots, n\}$; that is, partitions of $\{0,1, \ldots, n\}$ in which any element but the smallest one of each nonzero block can be barred. In the zero block (i.e., the one containing zero) no elements are barred.

For each element $\omega$ of the hyperoctahedral group $B_{n}$, we form a subposet $\Pi_{\omega}$ of $\Pi_{n}^{B}$ consisting of all signed partitions obtained by splitting the signed permutation $\omega$. In 
Figure 2 the subposet $\Pi_{\overline{2} 31}$ of $\Pi_{3}^{B}$ is shown. Just as for type $A$, it is clear that each subposet $\Pi_{\omega}$ determines a fundamental cycle $\rho_{\omega}$ in $\tilde{H}_{n-2}\left(\overline{\Pi_{n}^{B}}\right)$. It is not clear, however, that the elements $\rho_{\omega}, \omega \in B_{n}$, generate $\tilde{H}_{n-2}\left(\overline{\Pi_{n}^{B}}\right)$; nor is it clear how one would select cycles $\rho_{\omega}$ that form a basis for $\tilde{H}_{n-2}\left(\overline{\Pi_{n}^{B}}\right)$. Our geometric technique enables us to identify a basis whose elements are those $\rho_{\omega}$ for which the right-to-left maxima of $\omega$ are unbarred.

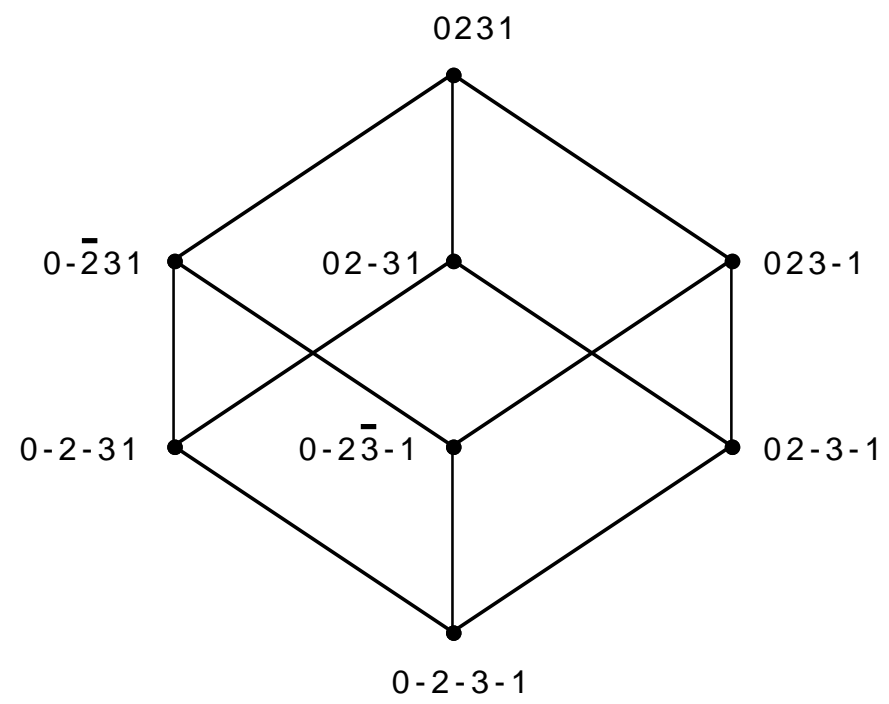

Figure 2

We will now give a somewhat more detailed description of the contents of the paper. The proper setting for our discussion is that of real hyperplane arrangements, or (even more generally) oriented matroids.

Let $\mathcal{A}$ be an arrangement of linear hyperplanes in $\mathbb{R}^{d}$. We assume that $\mathcal{A}$ is essential, meaning that $\bigcap \mathcal{A}:=\bigcap_{H \in \mathcal{A}} H=\{0\}$. The intersection lattice $L_{\mathcal{A}}$ is the family of intersections of subarrangements $\mathcal{A}^{\prime} \subseteq \mathcal{A}$, ordered by reverse inclusion. It is a geometric lattice, so it is known from a theorem of Folkman $[12]$ that $\widetilde{H}_{d-2}\left(\bar{L}_{\mathcal{A}}\right) \cong \mathbb{Z}^{\left|\mu_{L}(\hat{0}, \hat{1})\right|}$ and $\widetilde{H}_{i}\left(\bar{L}_{\mathcal{A}}\right)=0$ for all $i \neq d-2$, where $\bar{L}_{\mathcal{A}}=L_{\mathcal{A}}-\{\hat{0}, \hat{1}\}$. In fact, the order complex $\Delta\left(\bar{L}_{\mathcal{A}}\right)$ has the homotopy type of a wedge of $(d-2)$-spheres.

There are many copies of the Boolean lattice $2^{[d]}$ (or equivalently, the face lattice of the $(d-1)$-simplex) embedded in every geometric lattice of length $d$. Each such Boolean subposet determines a fundamental cycle in homology. In [3] Björner gives a combinatorial method for constructing homology bases using such Boolean cycles. This method, which in its simplest version is based on the so called "broken circuit" construction from matroid theory, is applicable to all geometric lattices (not only to intersection lattices of hyperplane arrangements). Although the cycles in the splitting basis are Boolean, the basis does not arise from the broken circuit construction. It turns out that the splitting basis does arise from the geometric construction in this paper.

There is a natural way to associate polytopal cycles in the intersection lattice $L_{\mathcal{A}}$ with regions of the arrangement $\mathcal{A}$. These cycles are not necessarily Boolean. They are 
fundamental cycles determined by face lattices of convex $(d-1)$-polytopes embedded in $L_{\mathcal{A}}$. We show that these cycles generate the homology of $\bar{L}_{\mathcal{A}}$. Moreover, we present a way of identifying those regions whose corresponding cycles form a basis. Here is a short and non-technical statement of the method.

Let $H$ be an affine hyperplane in $\mathbb{R}^{d}$ which is generic with respect to $\mathcal{A}$. The induced affine arrangement $\mathcal{A}_{H}=\{H \cap K \mid K \in \mathcal{A}\}$ in $H \cong \mathbb{R}^{d-1}$ will have certain regions that are bounded. Each bounded region $R$ is a convex $(d-1)$-polytope in $H$ and it is easy to see that a copy of its face lattice sits embedded in $L_{\mathcal{A}}$. Briefly, every face $F$ of $R$ is the intersection of the maximal faces containing it, and so $F$ can be mapped to the intersection of the linear spans (in $\mathbb{R}^{d}$ ) of these maximal faces, which is an element of $L_{\mathcal{A}}$. Thus, we have a cycle $\rho_{R} \in \widetilde{H}_{d-2}\left(\bar{L}_{\mathcal{A}}\right)$ for each bounded region $R$. A main result (Theorem 4.2) is that these cycles $\rho_{R}$, indexed by the bounded regions of $\mathcal{A}_{H}$, form a basis for $\widetilde{H}_{d-2}\left(\bar{L}_{\mathcal{A}}\right)$.

The regions of a Coxeter arrangement are simplicial cones that correspond bijectively to the elements of the Coxeter group. When the geometric method is applied to the intersection lattice of any Coxeter arrangement, the cycles in the resulting basis are Boolean and are indexed by the elements of the Coxeter group that correspond to the bounded regions of a generic affine slice. For type A, when the generic affine hyperplane $H$ is chosen appropriately one gets the splitting basis consisting of cycles $\rho_{\omega}$ indexed by the permutations $\omega$ that fix $n$. In Figure 3 the intersection of the Coxeter arrangement $\mathcal{A}_{3}$ with $H$ is shown. The bounded regions are labeled by their corresponding permutation.

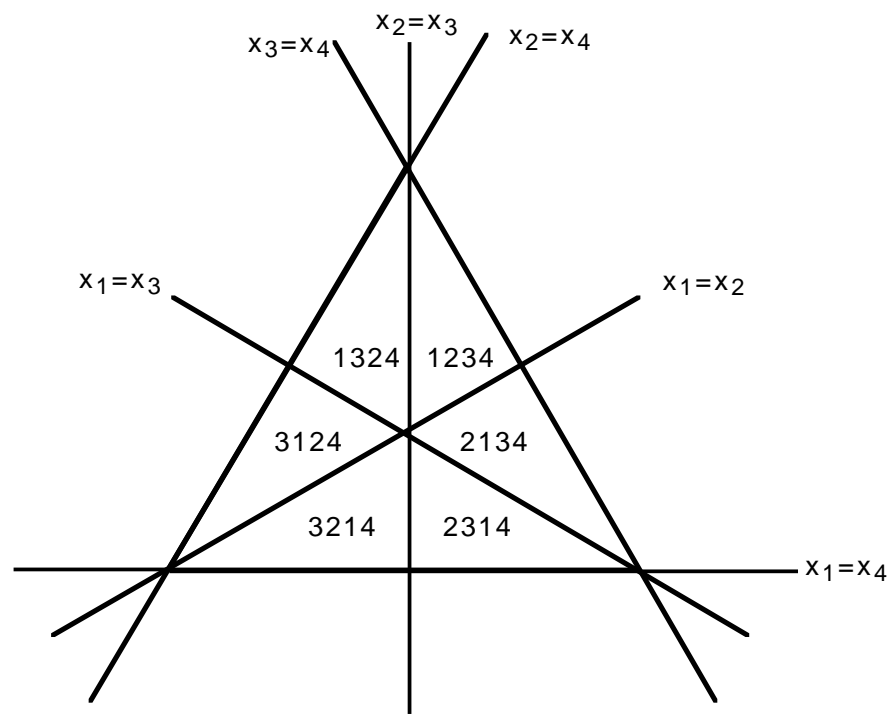

Figure 3

For type $\mathrm{B}$, when the generic affine hyperplane $H$ is chosen appropriately, one gets the type $B$ splitting basis consisting of cycles $\rho_{\omega}$ indexed by signed permutations $\omega$ whose right-to-left maxima are unbarred. The hyperplane arrangement $\mathcal{B}_{3}$ intersected with a 
cube is shown in Figure 4. The regions that have bounded intersection with $H$ are the ones that are labeled. The labels are the signed permutations whose right-to-left maxima are unbarred.

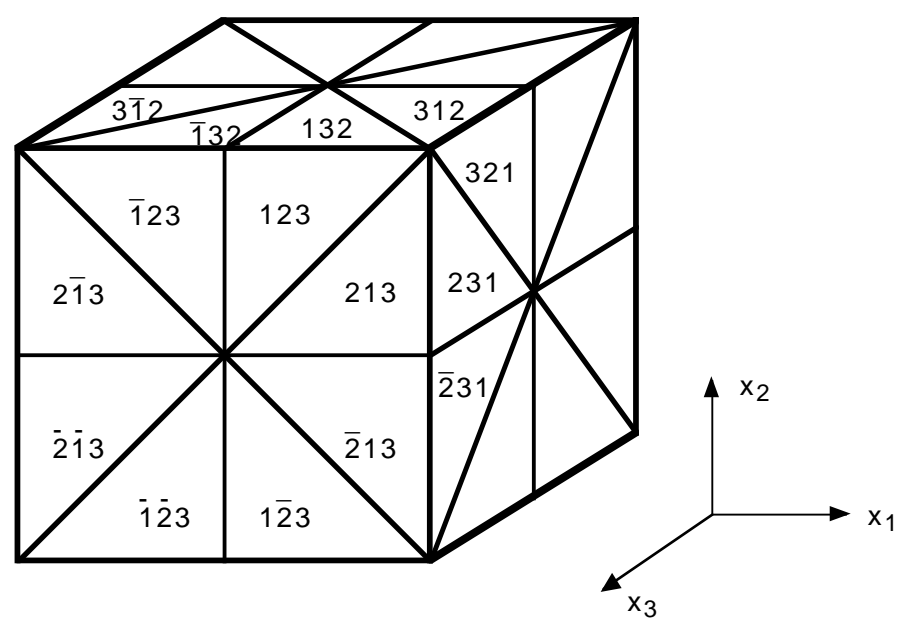

Figure 4

All arguments in the paper are combinatorial in nature, which means that they can be carried out for oriented matroids. So the construction of bases is applicable to geometric lattices of orientable matroids. Geometrically this means that we can allow some topological deformation of the hyperplane arrangements.

Major parts of this work (Sections 3, 4 and 6) were carried out at the Hebrew University in 1993 during the Jerusalem Combinatorics Conference. The rest was added in 1998. It has been brought to our attention that some of the material in Sections 3 and 4 shows similarities with work of others (see e.g. Proposition 5.6 of Damon [10] and parts of Ziegler [25], [26]); however, there is no substantial overlap or direct duplication.

\section{A lemma on shellable posets}

The concept of a shellable complex and a shellable poset will be considered known. See [6] for the definition and basic properties. In particular, we will make use of the shelling basis for homology and cohomology [6, Section 4]. A facet $F$ will be called a full restriction facet with respect to a shelling if $\mathcal{R}(F)=F$, where $\mathcal{R}(\cdot)$ is the restriction operator induced by the shelling. (Remark: Such facets were called homology facets in [6, Section 4].)

Our notation for posets is that of [6, Section 5]. For instance, if $P$ is a bounded poset with top element $\hat{1}$ and bottom element $\hat{0}$ then $\bar{P}$ denotes the proper part of $P$, which is defined to be $P \backslash\{\hat{0}, \hat{1}\}$; and if $P$ is an arbitrary poset then $\widehat{P}=P \uplus\{\hat{0}, \hat{1}\}$. Also, define $P_{<x}:=\{y \in P \mid y<x\}$ and $P_{\leq x}:=\{y \in P \mid y \leq x\}$.

The following simple lemma is a useful devise for identifying bases for homology of simplicial complexes. It is used implicitly in [20, proof of Theorem 2.2] and variations of 
it are used in $[7,8,13]$. For any element $\rho$ of the chain complex of a simplicial complex $\Delta$ and face $F$ of $\Delta$, we denote the coefficient of $F$ in $\rho$ by $\langle\rho, F\rangle$.

Lemma 2.1. Let $\Delta$ be a d-dimensional simplicial complex for which $\widetilde{H}_{d}(\Delta)$ has rank $t$. If $\rho_{1}, \rho_{2}, \ldots, \rho_{t}$ are $d$-cycles and $F_{1}, F_{2}, \ldots, F_{t}$ are facets such that the matrix $\left(\left\langle\rho_{i}, F_{j}\right\rangle\right)_{i, j \in[t]}$ is invertible over $\mathbb{Z}$, then $\rho_{1}, \rho_{2}, \ldots, \rho_{t}$ is a basis for $\widetilde{H}_{d}(\Delta)$.

Proof. Let $\sum_{i=1}^{t} a_{i} \rho_{i}=0$. Then

$$
\left(a_{1}, \ldots, a_{t}\right)\left(\left\langle\rho_{i}, F_{j}\right\rangle\right)_{i, j \in[t]}=(0, \ldots, 0)
$$

Since $\left(\left\langle\rho_{i}, F_{j}\right\rangle\right)_{i, j \in[t]}$ is invertible, $a_{i}=0$ for all $i$. Hence $\rho_{1}, \rho_{2}, \ldots, \rho_{t}$ are independent over $\mathbb{Q}$ as well as $\mathbb{Z}$. It follows that $\rho_{1}, \rho_{2}, \ldots, \rho_{t}$ forms a basis over $\mathbb{Q}$.

To see that $\rho_{1}, \rho_{2}, \ldots, \rho_{t}$ spans $\widetilde{H}_{d}(\Delta)$, let $\rho$ be a $d$-cycle. Then $\rho=\sum_{i=1}^{t} c_{i} \rho_{i}$ where $c_{i} \in \mathbb{Q}$. We have

$$
\left(c_{1}, \ldots, c_{t}\right)\left(\left\langle\rho_{i}, F_{j}\right\rangle\right)_{i, j \in[t]}=\left(\left\langle\rho, F_{1}\right\rangle, \ldots,\left\langle\rho, F_{t}\right\rangle\right)
$$

It follows that

$$
\left(c_{1}, \ldots, c_{t}\right)=\left(\left\langle\rho, F_{1}\right\rangle, \ldots,\left\langle\rho, F_{t}\right\rangle\right)\left(\left\langle\rho_{i}, F_{j}\right\rangle\right)_{i, j \in[t]}^{-1} \in \mathbb{Z}^{t}
$$

Hence $\rho$ is in the $\mathbb{Z}$-span of $\rho_{1}, \rho_{2}, \ldots, \rho_{t}$.

Suppose that $\Omega$ is a shelling order of the maximal chains of a pure shellable poset $P$ of length $r$. Let $M$ be the set of maximal elements of $P$. Recall the following two facts:

(i) For each $m \in M$, a shelling order $\Omega^{<m}$ is induced on the maximal chains of $P_{<m}$ by restricting $\Omega$ to the chains containing $m$ [2, Prop 4.2].

(ii) A shelling order $\Omega^{P \backslash M}$ is induced on the maximal chains of $P \backslash M$ as follows. Map each maximal chain $c$ in $P \backslash M$ to its $\Omega$-earliest extension $\varphi(c)=c \cup\{m\}, m \in M$. Note that $\varphi$ is injective. Now say that $c$ precedes $c^{\prime}$ in $\Omega^{P \backslash M}$ if and only if $\varphi(c)$ precedes $\varphi\left(c^{\prime}\right)[2$, Th. 4.1].

Let $\mathcal{F}\left(P_{<m}\right)$ and $\mathcal{F}(P \backslash M)$ denote the sets of full restriction facets induced by $\Omega^{<m}$ and $\Omega^{P \backslash M}$. Recall from [6, Section 4] that the shelling $\Omega^{<m}$ induces a basis $B\left(P_{<m}\right):=$ $\left\{\rho_{F}\right\}_{F \in \mathcal{F}\left(P_{<m}\right)}$ of $\widetilde{H}_{r}\left(P_{<m}\right)$ which is characterized by the property that $\left\langle\rho_{F}, F^{\prime}\right\rangle=\delta_{F, F^{\prime}}$ for all $F, F^{\prime} \in \mathcal{F}\left(P_{<m}\right)$.

Lemma 2.2. Let $P$ be a pure poset of length $r$ and $M$ the set of its maximal elements. Suppose that $P$ is shellable and acyclic. Then

(i) $\mathcal{F}(P \backslash M)=\biguplus_{m \in M} \mathcal{F}\left(P_{<m}\right)$,

(ii) $\biguplus_{m \in M} B\left(P_{<m}\right)$ is a basis for $\widetilde{H}_{r-1}(P \backslash M)$. 
Proof of (i). We claim that

$$
c \in \mathcal{F}\left(P_{<m}\right) \Longrightarrow \varphi(c)=c \cup\{m\} \text { and } c \in \mathcal{F}(P \backslash M) .
$$

Let $c \in \mathcal{F}\left(P_{<m}\right)$. This means that $c \backslash\{x\}$ is contained in an $\Omega^{<m}$-earlier maximal chain of $P_{<m}$, for every $x \in c$. If $\varphi(c)=c \cup\left\{m^{\prime}\right\}$ with $m^{\prime} \neq m$ then it would follow that $c \cup\{m\}$ is a full restriction facet of $P$, contradicting the assumption that $P$ is acyclic. Hence $\varphi(c)=c \cup\{m\}$. We can also conclude that $c \in \mathcal{F}(P \backslash M)$.

It follows from (1) that the sets $\mathcal{F}\left(P_{<m}\right), m \in M$, are disjoint and that

$$
\mathcal{F}(P \backslash M) \supseteq \biguplus_{m \in M} \mathcal{F}\left(P_{<m}\right) .
$$

The reverse inclusion will be a consequence of the following computations using the Möbius function $\mu(\hat{0}, x)$ of $\widehat{P}$. Since $P$ is acyclic we have that

$$
\sum_{x \in \widehat{P} \backslash\{\hat{1}\}} \mu(\hat{0}, x)=-\mu(\hat{0}, \hat{1})=-\widetilde{\chi}(P)=0 .
$$

Hence,

$$
\begin{aligned}
|\mathcal{F}(P \backslash M)| & =(-1)^{r} \sum_{x \in \hat{P} \backslash\{\hat{1}\} \backslash M} \mu(\hat{0}, x) \\
& =(-1)^{r-1} \sum_{m \in M} \mu(\hat{0}, m)=\sum_{m \in M}\left|\mathcal{F}\left(P_{<m}\right)\right| .
\end{aligned}
$$

Proof of (ii). For the homology basis of $\widetilde{H}_{r}(P \backslash M)$ we will use Lemma 2.1. Order $\mathcal{F}(P \backslash M)$ by $\Omega^{P \backslash M}$, and for each $c \in \mathcal{F}(P \backslash M)=\uplus_{m \in M} \mathcal{F}\left(P_{<m}\right)$, let $m_{c}$ be defined by $\varphi(c)=c \cup\left\{m_{c}\right\}$. By $(1), c \in \mathcal{F}\left(P_{<m_{c}}\right)$. Let $\rho_{c}$ be the element of $B\left(P_{<m_{c}}\right)$ corresponding to $c$. So, $\rho_{c}$ is the $(r-1)$-cycle in $P_{<m_{c}}$ with coefficient +1 at $c$ and coefficient 0 at all $c^{\prime} \in \mathcal{F}\left(P_{<m_{c}}\right) \backslash\{c\}$.

Suppose that $\rho_{c}$ has nonzero coefficient at some chain $c^{\prime} \neq c$. Since $c^{\prime}$ must come before $c$ in $\Omega^{<m}$ (the cycle $\rho_{c}$ has support on a subset of the chains in $P_{<m}$ that were present at the stage during the shelling $\Omega^{<m}$ when $c$ was introduced), it follows that $\varphi\left(c^{\prime}\right)$ precedes $\varphi(c)$ in $\Omega$, and hence that $c^{\prime}$ precedes $c$ in $\Omega^{P \backslash M}$. Hence the matrix $\left(\left\langle\rho_{c}, c^{\prime}\right\rangle\right)_{c, c^{\prime} \in \mathcal{F}(P \backslash M)}$ is lower triangular with 1's on the diagonal. It now follows from Lemma 2.1 that $\biguplus_{m \in M} B\left(P_{<m}\right)=\left\{\rho_{c}\right\}_{c \in \mathcal{F}(P \backslash M)}$ is a basis for $\widetilde{H}_{r}(P \backslash M)$.

\section{$3 \quad$ Affine hyperplane arrangements}

Let $\mathcal{A}=\left\{H_{1}, \ldots, H_{t}\right\}$ be an arrangement of affine (or linear) hyperplanes in $\mathbb{R}^{d}$. Each hyperplane $H_{i}$ divides $\mathbb{R}^{d}$ into three components: $H_{i}$ itself and the two connected components of $\mathbb{R}^{d} \backslash H_{i}$. For $x, y \in \mathbb{R}^{d}$, say that $x \equiv y$ if $x$ and $y$ are in the same component 
with respect to $H_{i}$, for all $i=1, \ldots, t$. This equivalence relation partitions $\mathbb{R}^{d}$ into open cells.

Let $P_{\mathcal{A}}$ denote the poset of cells (equivalence classes under $\equiv$ ), ordered by inclusion of their closures. $P_{\mathcal{A}}$ is called the face poset of $\mathcal{A}$. It is a finite pure poset with at most $d+1$ rank levels corresponding to the dimensions of the cells. The maximal elements of $P_{\mathcal{A}}$ are the regions of $\mathbb{R}^{d} \backslash \cup \mathcal{A}$. See Ziegler [25] for a detailed discussion of these facts.

Assume in what follows that the face poset $P_{\mathcal{A}}$ has length $d$. We will make use of the following technical properties of the order complex of $P_{\mathcal{A}}$.

\section{Proposition 3.1 ([25, Section 3]).}

(i) $P_{\mathcal{A}}$ is shellable.

(ii) $P_{\mathcal{A}}$ is homeomorphic to the d-ball.

(iii) Let $R$ be a region of $\mathbb{R}^{d} \backslash \bigcup \mathcal{A}$. Then

$$
\left(P_{\mathcal{A}}\right)_{<R} \cong \begin{cases}(d-1) \text {-sphere } & \text { if } R \text { is bounded } \\ (d-1) \text {-ball } & \text { otherwise. }\end{cases}
$$

If $R$ is a bounded region then its closure $\operatorname{cl}(R)$ is a convex $d$-polytope, and the open interval $\left(P_{\mathcal{A}}\right)_{<R}$ is the proper part of the face lattice of $\operatorname{cl}(R)$. The order complex of $\left(P_{\mathcal{A}}\right)_{<R}$, being a simplicial $(d-1)$-sphere, supports a unique (up to sign) fundamental $(d-1)$-cycle $\tau_{R}$.

Let $\overline{\bar{P}}_{\mathcal{A}}=\left\{\sigma \in P_{\mathcal{A}} \mid \operatorname{dim} \sigma<d\right\}$. Equivalently, $\overline{\bar{P}}_{\mathcal{A}}$ is the poset $P_{\mathcal{A}}$ with its maximal elements (the regions) removed. Also, let $\mathcal{B}=\{$ bounded regions $\}$.

\section{Proposition 3.2.}

(i) $\overline{\bar{P}}_{\mathcal{A}}$ has the homotopy type of a wedge of $(d-1)$-spheres.

(ii) $\left\{\tau_{R}\right\}_{R \in \mathcal{B}}$ is a basis for $\widetilde{H}_{d-1}\left(\overline{\bar{P}}_{\mathcal{A}}\right)$.

Proof. Part (i) follows from the fact that shellability is preserved by rank-selection [2, Th. 4.1], and that a shellable pure $(d-1)$-complex has the stated homotopy type. Since $\left\{\tau_{R}\right\}$ is (due to uniqueness) the shelling basis for $\widetilde{H}_{d-1}\left(\left(P_{\mathcal{A}}\right)_{<R}\right)$ when $R \in \mathcal{B}$, and $\widetilde{H}_{d-1}\left(\left(P_{\mathcal{A}}\right)_{<R}\right)=$ 0 when $R \notin \mathcal{B}$, part (ii) follows from Lemma 2.2 .

Remark 3.3. From Proposition 3.2 one can deduce the fact that the union of all hyperplanes of an affine arrangement is homotopy equivalent to a wedge of $(d-1)$-spheres, the number of spheres being equal to the number of bounded regions of the complement. Furthermore, the boundaries of the bounded regions induce spherical cycles that form a basis for $\widetilde{H}_{d-1}\left(\mathbb{R}^{d} \backslash \bigcup \mathcal{A}\right)$. 
Let $L_{\mathcal{A}}$ denote the intersection semilattice of $\mathcal{A}$. Its elements are the nonempty intersections $\bigcap \mathcal{A}^{\prime}$ of subfamilies $\mathcal{A}^{\prime} \subseteq \mathcal{A}$, and the order relation is reverse inclusion. $L_{\mathcal{A}}$ is a pure poset of length $d$. Its unique minimal element is $\mathbb{R}^{d}$ (corresponding to $\mathcal{A}^{\prime}=\varnothing$ ), which (according to convention) will be denoted by $\hat{0}$. The minimal elements of $L_{\mathcal{A}} \backslash\{\hat{0}\}$ are the hyperplanes $H_{i} \in \mathcal{A}$, and the maximal elements are the single points of $\mathbb{R}^{d}$ obtainable as intersections of subfamilies $\mathcal{A}^{\prime} \subseteq \mathcal{A}$. $L_{\mathcal{A}}$ is a geometric semilattice in the sense of $[22]$.

For each cell $\sigma \in P_{\mathcal{A}}$, let $z(\sigma)$ be the affine span of $\sigma$. The subspace $z(\sigma)$ can also be described as follows. By definition, $\sigma$ is the intersection of certain hyperplanes in $\mathcal{A}$ (call the set of these hyperplanes $\left.\mathcal{A}_{\sigma}\right)$ and certain halfspaces determined by other hyperplanes in $\mathcal{A}$. Then, $z(\sigma)=\bigcap \mathcal{A}_{\sigma}$. This shows that $\operatorname{dim} \sigma=\operatorname{dim} z(\sigma)$ and that $z(\sigma) \in L_{\mathcal{A}}$. The map

$$
z: P_{\mathcal{A}} \rightarrow L_{\mathcal{A}}
$$

is clearly order-reversing, and it restricts to an order-reversing map

$$
z: \overline{\bar{P}}_{\mathcal{A}} \rightarrow L_{\mathcal{A}} \backslash\{\hat{0}\}
$$

In various versions, the following result appears in several places in the literature; see the discussion following Lemma 3.2 of [26].

Proposition 3.4. The map $z: \overline{\bar{P}}_{\mathcal{A}} \rightarrow L_{\mathcal{A}} \backslash\{\hat{0}\}$ induces homotopy equivalence of order complexes.

Proof. We will use the Quillen fiber lemma [18]. This reduces the question to checking that every fiber $z^{-1}\left(\left(L_{\mathcal{A}}\right)_{\geq x}\right)$ is contractible, $x \in L_{\mathcal{A}} \backslash\{\hat{0}\}$. But by Proposition 3.1 (ii) such a fiber is homeomorphic to a $\operatorname{dim}(x)$-ball, so we are done.

The simplicial map $z$ induces a homomorphism

$$
z_{*}: \widetilde{H}_{d-1}\left(\overline{\bar{P}}_{\mathcal{A}}\right) \rightarrow \widetilde{H}_{d-1}\left(L_{\mathcal{A}} \backslash\{\hat{0}\}\right),
$$

which (as a consequence of Proposition 3.4) is an isomorphism. The following is an immediate consequence of Propositions 3.2 and 3.4.

Theorem 3.5. $\left\{z_{*}\left(\tau_{R}\right)\right\}_{R \in \mathcal{B}}$ is a basis of $\widetilde{H}_{d-1}\left(L_{\mathcal{A}} \backslash\{\hat{0}\}\right)$.

Recall that $\tau_{R}$ is the fundamental cycle of the proper part of the face lattice of the convex polytope $\operatorname{cl}(R)$, for each bounded region $R$. Since the map $z$ is injective on each lower interval $\left(P_{\mathcal{A}}\right)_{<R}$ it follows that the cycles $z_{*}\left(\tau_{R}\right)$ are also "polytopal", arising from copies of the proper part of the dual face lattice of $\operatorname{cl}(R)$ embedded in $L_{\mathcal{A}}$.

Remark 3.6. It is a consequence of Theorem 3.5 that

$$
\operatorname{rank} \widetilde{H}_{d-1}\left(L_{\mathcal{A}} \backslash\{\hat{0}\}\right)=\operatorname{card} \mathcal{B} .
$$


This enumerative corollary is equivalent to the following result of Zaslavsky [23]:

$$
\operatorname{card} \mathcal{B}=\left|\sum_{x \in L_{\mathcal{A}}} \mu(\hat{0}, x)\right|
$$

Indeed, we have that

$$
\operatorname{rank} \widetilde{H}_{d-1}\left(L_{\mathcal{A}} \backslash\{\hat{0}\}\right)=\left|\mu_{L_{\mathcal{A}} \cup\{\hat{1}\}}(\hat{0}, \hat{1})\right|,
$$

since $L_{\mathcal{A}} \cup\{\hat{1}\}$ is the intersection lattice of a central arrangement and is hence a geometric lattice. Since

$$
\mu_{L_{\mathcal{A}} \cup\{\hat{1}\}}(\hat{0}, \hat{1})=-\sum_{x \in L_{\mathcal{A}}} \mu(\hat{0}, x),
$$

the results are equivalent.

Remark 3.7. Our work in this section has the purpose to provide a short but exact route to the results of the following section, in particular to Theorem 4.2. In the process, a natural method for constructing bases for geometric semilattices that are intersection lattices of real affine hyperplane arrangements is given by Theorem 3.5. For general geometric semilattices, a method for constructing bases which generalizes the broken circuit construction of [3] is given by Ziegler [26]. This construction does not reduce to the construction given by Theorem 3.5 in the case that the geometric semilattice is the intersection lattice of a real affine hyperplane arrangement.

\section{Central hyperplane arrangements}

Let $\mathcal{A}$ be an essential arrangement of linear hyperplanes in $\mathbb{R}^{d}$. As before, let $L_{\mathcal{A}}$ denote the set of intersections $\bigcap \mathcal{A}^{\prime}$ of subfamilies $\mathcal{A}^{\prime} \subseteq \mathcal{A}$ (such intersections are necessarily nonempty in this case) partially ordered by reverse inclusion. The finite lattice $L_{\mathcal{A}}$ is called the intersection lattice of $\mathcal{A}$. It is a geometric lattice of length $d$.

Now, let $H$ be an affine hyperplane in $\mathbb{R}^{d}$ which is generic with respect to $\mathcal{A}$. Genericity here means that $\operatorname{dim}(H \cap X)=\operatorname{dim}(X)-1$ for all $X \in L_{\mathcal{A}}$. Equivalently, $0 \notin H$ and $H \cap X \neq \varnothing$ for all 1-dimensional subspaces $X \in L_{\mathcal{A}}$.

Let $\mathcal{A}_{H}=\{H \cap K \mid K \in \mathcal{A}\}$. This is an affine hyperplane arrangement induced in $H \cong \mathbb{R}^{d-1}$. We denote by $L_{\mathcal{A}_{H}}$ its intersection semilattice.

Lemma 4.1. $L_{\mathcal{A}_{H}} \cong L_{\mathcal{A}} \backslash\{\hat{1}\}$.

Proof. The top element $\hat{1}$ of $L_{\mathcal{A}}$ is the 0 -dimensional subspace $\{0\}$ of $\mathbb{R}^{d}$. Thus $X \mapsto$ $H \cap X$ defines an order-preserving map $L_{\mathcal{A}} \backslash\{\hat{1}\} \rightarrow L_{\mathcal{A}_{H}}$, which is easily seen to be an isomorphism.

The connected components of $\mathbb{R}^{d} \backslash \cup \mathcal{A}$ are pointed open convex polyhedral cones, that we call regions. Although none of these regions is bounded (since $\mathcal{A}$ is central), each region $R$, nevertheless, induces a cycle $\rho_{R}$ in $\widetilde{H}_{d-2}\left(\bar{L}_{\mathcal{A}}\right)$ as follows. Let $P_{R}$ denote the face 
lattice of the closed cone $\operatorname{cl}(R)$. That is, $P_{R}$ is the lower interval $\left(P_{\mathcal{A}}\right)_{\leq R}$. Clearly $P_{R}$ is isomorphic to the face lattice of the convex polytope $\operatorname{cl}(R \cap M)$, where $M$ is any affine hyperplane such that $R \cap M$ is nonempty and bounded. The map $z: P_{\mathcal{A}} \rightarrow L_{\mathcal{A}}$ defined in Section 3 clearly embeds a copy of the dual of $P_{R}$ in $L_{\mathcal{A}}$. Hence the image $z\left(P_{R}\right)$ is a subposet of $L_{\mathcal{A}}$ whose proper part is $(d-2)$ )-spherical (meaning that its order complex is homeomorphic to $S^{d-2}$ ). Let $\rho_{R}$ be the fundamental cycle (uniquely defined up to sign) of the proper part of the subposet $z\left(P_{R}\right)$.

Theorem 4.2. Let $\mathcal{A}$ be a central and essential hyperplane arrangement in $\mathbb{R}^{d}$ and let $H$ be an affine hyperplane, generic with respect to $\mathcal{A}$. Then the collection of cycles $\rho_{R}$ corresponding to regions $R$ such that $R \cap H$ is nonempty and bounded, form a basis of $\widetilde{H}_{d-2}\left(\bar{L}_{\mathcal{A}}\right)$.

Proof. This follows immediately from Theorem 3.5 and the fact that $\bar{L}_{\mathcal{A}} \cong L_{\mathcal{A}_{H}} \backslash\{\hat{0}\}$ (Lemma 4.1).

In order to apply Theorem 4.2 to the examples given in subsequent sections we will need to choose an appropriate generic affine hyperplane and determine the regions whose affine slices are bounded. The following lemma provides a useful way of doing this.

Lemma 4.3. Let $\mathcal{A}$ be a central and essential hyperplane arrangement in $\mathbb{R}^{d}$. Suppose $\mathbf{v}$ is a nonzero element of $\mathbb{R}^{d}$ such that the affine hyperplane $H_{\mathbf{v}}$ through $\mathbf{v}$ and normal to $\mathbf{v}$, is generic with respect to $\mathcal{A}$. Then for any region $R$ of $\mathcal{A}, R \cap H_{\mathbf{v}}$ is nonempty and bounded if and only if $\mathbf{v} \cdot \mathbf{x}>0$ for all $\mathbf{x} \in R$.

Proof. $(\Rightarrow)$ Suppose $R \cap H_{\mathbf{v}}$ is nonempty and bounded. It is not difficult to see that if an affine slice of a cone is nonempty and bounded, then the cone is a cone over the affine slice. Hence $R$ is a cone over $R \cap H_{\mathbf{v}}$. That is, every element of $R$ is a positive scalar multiple of an element of $R \cap H_{\mathbf{v}}$. It follows that since $\mathbf{v} \cdot \mathbf{x}>0$ for all $\mathbf{x} \in H_{\mathbf{v}}, \mathbf{v} \cdot \mathbf{x}>0$ for all $\mathrm{x} \in R$.

$(\Leftarrow)$ Suppose $R \cap H_{\mathbf{v}}$ is empty or unbounded. If the former holds then $\mathbf{v} \cdot \mathbf{x} \leq 0$ for all $\mathbf{x} \in R$. Indeed, if $\mathbf{v} \cdot \mathbf{x}>0$ for some $\mathbf{x} \in R$ then $\frac{\mathbf{v} \cdot \mathbf{v}}{\mathbf{v} \cdot \mathbf{x}} \mathbf{x} \in R \cap H_{\mathbf{v}}$.

We now assume $R \cap H_{\mathbf{v}}$ is unbounded. Then there is a sequence of points $\mathbf{x}_{1}, \mathbf{x}_{2} \ldots$ in $R \cap H_{\mathbf{v}}$ whose distance from the origin goes to infinity. Let $\mathbf{e}_{i}$ be the unit vector in the direction of the vector $\mathbf{x}_{i}$. Each $\mathbf{e}_{i}$ is in the intersection of $\mathrm{R}$ and the unit sphere centered at the origin. Hence, by passing to a subsequence if necessary, we can assume that the sequence of $\mathbf{e}_{i}$ 's converge to a unit vector $\mathbf{e}$ in the closure of $R$. Since the cosine of the angle between $\mathbf{e}_{i}$ and $\mathbf{v}$ is $\frac{\|\mathbf{v}\|}{\left\|\mathbf{x}_{i}\right\|}$, the cosine of the angles approach 0 . Hence the cosine of the angle between $\mathbf{e}$ and $\mathbf{v}$ is 0 , or equivalently $\mathbf{v} \cdot \mathbf{e}=0$.

Since $\mathbf{e}$ is in the closure of $R$, either $\mathbf{e} \in R$ or there is a unique face $F$ of $R$ such that $\mathbf{e}$ is in the interior of $F$. If $\mathbf{e} \in R$ we are done. So suppose $\mathbf{e}$ is in the interior of the face $F$. If $\mathbf{v} \cdot \mathbf{x}=0$ for all $\mathbf{x} \in F$ then the linear span of $F$ is an intersection of hyperplanes contained in the linear hyperplane with normal vector $\mathbf{v}$. This contradicts the genericity of $H_{\mathbf{v}}$. It follows that $\mathbf{v} \cdot \mathbf{x} \neq 0$ for some $\mathbf{x} \in F$. If $\mathbf{v} \cdot \mathbf{x}<0$ then there is a point $\mathbf{y} \in R$ that is close enough to $\mathbf{x}$ so that $\mathbf{v} \cdot \mathbf{y}<0$ and we are done. If $\mathbf{v} \cdot \mathbf{x}>0$ then consider the 
point $\mathbf{e}-a \mathbf{x}$ where $a>0$. We have $\mathbf{v} \cdot(\mathbf{e}-a \mathbf{x})=-a(\mathbf{v} \cdot \mathbf{x})<0$. By choosing $a$ to be small enough, we insure that the point $\mathbf{e}-a \mathbf{x}$ is close enough to $\mathbf{e}$ to be in $F$, since $\mathbf{e}$ is in the interior of $F$. Hence we have a point in $F$ whose dot product with $\mathbf{v}$ is negative, putting us back in the previous case.

Remark 4.4. Theorem 4.2 can be extended to a geometric construction of bases for the Whitney homology (or equivalently the Orlik-Solomon algebra, see e.g. [4, Sect. 10]) of the intersection lattice of a real central hyperplane arrangement. This involves the definition of a vector $v$ being totally generic with respect to the arrangement. Since we will not pursue this direction we omit further mention of it.

\section{Oriented matroids}

The arguments and results of the previous two sections can be generalized to oriented matroids. This generalization will be outlined in this section. The treatment here will be sketchy and can be skipped with no loss of continuity. The basics of oriented matroid theory will be assumed to be known. We refer to [5] for all definitions and notation.

Let $(\mathcal{L}, E, g)$ be an affine oriented matroid of rank $r$ and with affine face lattice $\mathcal{L}^{+}=\left\{X \in \mathcal{L} \mid X_{g}=+\right\}$, cf. [5, Section 4.5]. The maximal elements of $\mathcal{L}^{+}$are the topes, corresponding to regions in the realizable case. Let $\mathcal{L}^{++}$be the bounded complex (a subcomplex of $\mathcal{L}^{+}$), and let $\mathcal{B}^{++}$be the set of bounded topes, i.e., $\mathcal{B}^{++}=\left\{X \in \mathcal{L}^{++} \mid\right.$ $\operatorname{rank}(X)=r-1\}$.

We have from [5, Th. 4.5.7] that $\mathcal{L}^{+}$is a shellable ball. Furthermore, if $T \in \mathcal{B}^{++}$then the order complex of the open interval $(0, T)$ in $\mathcal{L}^{+}$is homeomorphic to $S^{r-2}$ [5, Cor. 4.3.7]. Therefore, each $T \in \mathcal{B}^{++}$induces a spherical fundamental cycle $\tau_{T}$ in $\widetilde{H}_{r-2}\left(\overline{\overline{\mathcal{L}^{+}}}\right)$, where $\overline{\overline{\mathcal{L}^{+}}}=\mathcal{L}^{+} \backslash\{$ topes $\}$.

\section{Proposition 5.1.}

(i) $\overline{\overline{\mathcal{L}^{+}}}$has the homotopy type of a wedge of $\left|\mathcal{B}^{++}\right|$copies of the $(r-2)$-sphere.

(ii) $\left\{\tau_{T}\right\}_{T \in \mathcal{B}^{++}}$is a basis of $\widetilde{H}_{r-2}\left(\overline{\overline{\mathcal{L}^{+}}}\right)$.

Proof. The proof of Proposition 3.2 generalizes.

Now, let $L$ be the intersection lattice (or "lattice of flats") of the oriented matroid $\mathcal{L}$, and let $z: \mathcal{L} \rightarrow L$ be the "zero map" [5, Prop. 4.1.13]. Furthermore, let $L^{g}:=\{x \in$ $L \mid x \nsupseteq g\}=L \backslash[g, \hat{1}]$. This is a geometric semilattice. The zero map restricts to an order-reversing surjection $z: \mathcal{L}^{+} \rightarrow L^{g}$, and further to a surjection $z: \overline{\overline{\mathcal{L}^{+}}} \rightarrow L^{g} \backslash\{\hat{0}\}$.

Proposition 5.2. The map $z: \overline{\overline{\mathcal{L}^{+}}} \rightarrow L^{g} \backslash\{\hat{0}\}$ induces homotopy equivalence of order complexes.

Proof. The proof of Proposition 3.4 generalizes. Here one uses that the Quillen fibers $z^{-1}\left(\left(L^{g}\right)_{\geq x}\right), x \neq \hat{0}$, are balls by [5, Th. 4.5.7], and hence contractible. 
The restriction of $z$ to an open interval $(0, T)$ in $\mathcal{L}^{+}$, with $T \in \mathcal{B}^{++}$, gives an isomorphism of $(0, T)$ onto its image in $L^{g}$. This image is a subposet of $L^{g} \backslash\{\hat{0}\}$ homeomorphic to the $(r-2)$-sphere. Let $\rho_{T} \in \widetilde{H}_{r-2}\left(L^{g} \backslash\{\hat{0}\}\right)$ be the corresponding fundamental cycle.

\section{Theorem 5.3.}

(i) $L^{g} \backslash\{\hat{0}\}$ has the homotopy type of $\left|\mathcal{B}^{++}\right|$copies of the $(r-2)$-sphere.

(ii) $\left\{\rho_{T}\right\}_{T \in \mathcal{B}^{++}}$is a basis of $\widetilde{H}_{r-2}\left(L^{g} \backslash\{\hat{0}\}\right)$.

Proof. This follows from Propositions 5.1 and 5.2, since

$$
z_{*}: \widetilde{H}_{r-2}\left(\overline{\overline{\mathcal{L}^{+}}}\right) \rightarrow \widetilde{H}_{r-2}\left(L^{g} \backslash\{\hat{0}\}\right)
$$

is an isomorphism and $z_{*}\left(\tau_{T}\right)=\rho_{T}$.

The treatment of affine oriented matroids so far parallels that of affine hyperplane arrangements in Section 3. We will now move on to the oriented matroid version of the material in Section 4.

Let $\mathcal{L} \subseteq\{+,-, 0\}^{E}$ be an oriented matroid of rank $r$, and let $z: \mathcal{L} \rightarrow L$ be the zero map to the corresponding intersection lattice $L$. Let $\left.\mathcal{L}_{g} \subseteq\{+,-, 0\}\right\}^{E \uplus g}$ be an extension of $\mathcal{L}$ by a generic element $g \notin E$. Genericity here means that $g \notin \operatorname{span} A$ for every $A \subseteq E$ with $\operatorname{rank}(A)<r$, cf. [5, Sect. 7.1].

Consider the affine oriented matroid $\left(\mathcal{L}_{g}, E \uplus g, g\right)$ and let $L_{g}$ be its intersection semilattice. We have that $z\left(\mathcal{L}_{g}\right)=L_{g}$ and $z\left(\mathcal{L}_{g}^{+}\right)=\left(L_{g}\right)^{g}$.

Lemma 5.4. $\left(L_{g}\right)^{g} \cong L \backslash\{\hat{1}\}$.

Proof. This analog of Lemma 4.1 is clear. It is basically a reformulation of the definition of genericity.

Let $\mathcal{B}^{++}$be the bounded topes of $\mathcal{L}_{g}$ (with respect to $g$ ). Because of the isomorphism

$$
\left(L_{g}\right)^{g} \backslash\{\hat{0}\} \cong \bar{L}:=L \backslash\{\hat{0}, \hat{1}\},
$$

we get cycles $\rho_{T} \in \widetilde{H}_{r-2}(\bar{L})$ as before.

\section{Theorem 5.5.}

(i) $\bar{L}$ has the homotopy type of a wedge of $\left|\mathcal{B}^{++}\right|$copies of the $(r-2)$-sphere.

(ii) $\left\{\rho_{T}\right\}_{T \in \mathcal{B}^{+}}$is a basis for $\widetilde{H}_{r-2}(\bar{L})$.

Proof. This follows from Theorem 5.3 and Lemma 5.4.

The theorem gives a geometric method for constructing a basis for the homology of the geometric lattice of any orientable matroid. Note that to define the set $\mathcal{B}^{++}$, and hence the basis, we must make a generic extension of $\mathcal{L}$. Different extensions will yield different bases. 


\section{Type $A$ : The braid arrangement}

The hyperplane arrangement $\mathcal{A}_{n-1}=\left\{H_{i j}: 1 \leq i<j \leq n\right\}$ in $\mathbb{R}^{n}$, where $H_{i j}=\left\{\mathbf{x} \in \mathbb{R}^{n}\right.$ : $\left.x_{i}=x_{j}\right\}$, is known as the braid arrangement or the type $A$ Coxeter arrangement. The orthogonal reflection $\sigma_{i j}$ across the hyperplane $H_{i j}$ acts on $\left(x_{1}, \ldots, x_{n}\right) \in \mathbb{R}^{n}$ by switching its $i$ th and $j$ th coordinates. These reflections generate the symmetric group $S_{n}$ acting on $\mathbb{R}^{n}$ by permuting coordinates.

The braid arrangement is not essential. To make it essential let

$$
K=\left\{\mathbf{x} \in \mathbb{R}^{n}: x_{1}+\cdots+x_{n}=0\right\}
$$

and define

$$
\mathcal{A}_{n-1}^{\prime}=\left\{H_{i j}^{\prime}=K \cap H_{i j}: 1 \leq i<j \leq n\right\} .
$$

Then $\mathcal{A}_{n-1}^{\prime}$ is an essential central hyperplane arrangement in the $(n-1)$-dimensional space $K$. It is clear that the intersection lattices $L_{\mathcal{A}_{n-1}}$ and $L_{\mathcal{A}_{n-1}^{\prime}}$ are isomorphic. They are also isomorphic to the partition lattice $\Pi_{n}$. Indeed, for each $\pi \in \Pi_{n}$, let $\ell_{\pi}$ be the linear subspace of $\mathbb{R}^{n}$ consisting of all points $\left(x_{1}, \ldots, x_{n}\right)$ such that $x_{i}=x_{j}$ whenever $i$ and $j$ are in the same block of $\pi$. The map $\pi \mapsto \ell_{\pi} \cap K$ is an isomorphism from $\Pi_{n}$ to $L_{\mathcal{A}_{n-1}^{\prime}}$. Let $\gamma$ denote the inverse of this isomorphism.

The arrangement $\mathcal{A}_{n-1}^{\prime}$ has $n$ ! regions which are all simplicial cones and are in a natural one-to-one correspondence with the elements of the associated Coxeter group $S_{n}$. Under this correspondence a permutation $\omega \in S_{n}$ corresponds to the region

$$
R_{\omega}=\left\{\mathbf{x} \in K: x_{\omega(1)}<x_{\omega(2)}<\cdots<x_{\omega(n)}\right\} .
$$

Consider the cycle $\rho_{R_{\omega}} \in \widetilde{H}_{n-3}\left(\bar{L}_{A_{n-1}^{\prime}}\right)$ whose general construction was given in Section 4 . We now give a simple explicit description of the image of $\rho_{R_{\omega}}$ in $\widetilde{H}_{n-3}\left(\Pi_{n}\right)$ under the isomorphism $\gamma$.

To split a permutation $\omega \in S_{n}$ at positions $i_{1}<\cdots<i_{k}$ in $[n-1]$ is to form the partition with $k+1$ blocks,

$$
\left\{\omega(1), \ldots, \omega\left(i_{1}\right)\right\},\left\{\omega\left(i_{1}+1\right), \ldots, \omega\left(i_{2}\right)\right\}, \ldots,\left\{\omega\left(i_{k}+1\right), \ldots, \omega(n)\right\} .
$$

(To split $\omega$ at the empty set of positions is to form the partition with one block.) Let $\Pi_{\omega}$ denote the induced subposet of $\Pi_{n}$ consisting of all partitions obtained by splitting the permutation $\omega$. Clearly $\Pi_{\omega}$ is isomorphic to the lattice of subsets of $[n-1]$. Hence $\bar{\Pi}_{\omega}$ is spherical.

Proposition 6.1. For all $\omega \in S_{n}$, the image $\gamma\left(\rho_{R_{\omega}}\right)$ is the fundamental cycle of $\bar{\Pi}_{\omega}$.

Proof. Recall the map $z: P_{\mathcal{A}_{n-1}^{\prime}} \rightarrow L_{\mathcal{A}_{n-1}^{\prime}}$ that takes cells to their affine span (defined in Section 3). We show that $\gamma$ restricts to an isomorphism from the subposet $z\left(P_{R_{\omega}}\right)$ of $L_{A_{n-1}^{\prime}}$ to $\Pi_{\omega}$. Observe that elements of the face lattice $P_{R_{\omega}}$ are sets of the form

$$
\begin{array}{r}
\left\{\mathbf{x} \in K: x_{\omega(1)}=\cdots=x_{\omega\left(i_{1}\right)}<x_{\omega\left(i_{1}+1\right)}=\cdots=x_{\omega\left(i_{2}\right)}<\cdots\right. \\
\left.\cdots<x_{\omega\left(i_{k}+1\right)}=\cdots=x_{\omega(n)}\right\}, \\
\end{array}
$$


where $1 \leq i_{1}<\cdots<i_{k} \leq n-1$. The linear span of such a set is the subspace

$$
\begin{array}{r}
\left\{\mathbf{x} \in K: x_{\omega(1)}=\cdots=x_{\omega\left(i_{1}\right)}, x_{\omega\left(i_{1}+1\right)}=\cdots=x_{\omega\left(i_{2}\right)}, \ldots\right. \\
\left.\ldots, x_{\omega\left(i_{k}+1\right)}=\cdots=x_{\omega(n)}\right\} .
\end{array}
$$

Hence $z\left(P_{R_{\omega}}\right)$ is the poset of subspaces of the form given in (3) ordered by reverse inclusion. Clearly $\gamma$ takes the subspace given in (3) to the partition given in (2).

We now choose a vector $\mathbf{v}$ in $K$ that satisfies the hypothesis of Lemma 4.3 and use Lemma 4.3 to describe the permutations $\omega \in S_{n}$ for which the regions $R_{\omega} \cap H_{\mathbf{v}}$ are bounded.

Proposition 6.2. Let $\mathbf{v}=(-1,-1, \ldots,-1, n-1) \in K$. Then the affine hyperplane $H_{\mathbf{v}} \cap K$ is generic with respect to the arrangement $\mathcal{A}_{n-1}^{\prime}$ of $K$. Moreover, for all $\omega \in S_{n}$, $R_{\omega} \cap H_{\mathbf{v}}$ is bounded if and only if $\omega(n)=n$.

Proof. Recall that genericity is equivalent to the condition that for all 1-dimensional subspaces $X \in L_{\mathcal{A}_{n-1}^{\prime}}, H_{\mathbf{v}} \cap X \neq \emptyset$. The 1-dimensional intersections of hyperplanes in $\mathcal{A}_{n-1}^{\prime}$ have the form

$$
X=\left\{\mathbf{x} \in K: x_{i_{1}}=x_{i_{2}}=\cdots=x_{i_{k}}, x_{i_{k+1}}=x_{i_{k+2}}=\cdots=x_{i_{n-1}}=x_{n}\right\},
$$

where $1 \leq k \leq n-1$ and $\left\{\left\{i_{1}, i_{2}, \ldots, i_{k}\right\},\left\{i_{k+1}, \ldots, i_{n-1}, n\right\}\right\}$ is a partition of $[n]$. Let $\mathbf{x} \in \mathbb{R}^{n}$ be defined by

$$
x_{i_{j}}=\left\{\begin{array}{cl}
-\frac{(n-k)(n-1)}{k} & j=1, \ldots k \\
n-1 & j=k+1, \ldots, n-1
\end{array}\right.
$$

and $x_{n}=n-1$. One can easily check that $\mathbf{x} \in H_{\mathbf{v}} \cap X$. Hence $H_{\mathbf{v}} \cap K$ is generic.

To prove that $R_{\omega} \cap H_{\mathbf{v}}$ is bounded if and only if $\omega(n)=n$, we apply Lemma 4.3. Note that for all $\mathbf{x} \in K$,

$$
\mathbf{v} \cdot \mathbf{x}=n x_{n} .
$$

Suppose $\omega(n)=n$. For all $\mathbf{x} \in R_{\omega}$, we have $x_{\omega(1)}<\cdots<x_{\omega(n)}$ and $\sum_{i=1}^{n} x_{i}=0$. Hence $x_{\omega(n)}>0$. It follows that $\mathbf{v} \cdot \mathbf{x}=n x_{\omega(n)}>0$ for all $\mathbf{x} \in R_{\omega}$. By Lemma $4.3 R_{\omega} \cap H_{\mathbf{v}}$ is bounded. Now suppose $\omega(n) \neq n$. Clearly there exists an $\mathbf{x} \in R_{\omega}$ such that $x_{\omega(i)}<0$ for all $i=1, \ldots, n-1$. For such an $\mathbf{x}$, we have $\mathbf{v} \cdot \mathbf{x}=n x_{n}<0$. We conclude by Lemma 4.3 that $R_{\omega} \cap H_{\mathbf{v}}$ is not bounded.

Theorem 6.3 (Splitting basis [20]). For $\omega \in S_{n}$, let $\rho_{\omega}$ be the fundamental cycle of $\bar{\Pi}_{\omega}$. Then

$$
\left\{\rho_{\omega}: \omega \in S_{n} \text { and } \omega(n)=n\right\}
$$

is a basis for $\widetilde{H}_{n-3}\left(\bar{\Pi}_{n}\right)$.

Proof. This follows from Theorem 4.2 and Propositions 6.1 and 6.2. 


\section{$7 \quad$ Type $B$}

The type $B$ Coxeter arrangement is the hyperplane arrangement

$$
\begin{aligned}
\mathcal{B}_{n}= & \left\{x_{i}=x_{j}: 1 \leq i<j \leq n\right\} \cup\left\{x_{i}=-x_{j}: 1 \leq i<j \leq n\right\} \cup \\
& \left\{x_{i}=0: 1 \leq i \leq n\right\} .
\end{aligned}
$$

The orthogonal reflections across the hyperplanes generate the hyperoctahedral group $B_{n}$. We will view the elements of $B_{n}$ as signed permutations, that is, words consisting of $n$ distinct letters from $[n]$ where any of the letters can have a bar placed above it. It will also be convenient to express elements of $B_{n}$ as pairs $(\omega, \epsilon)$ where $\omega \in S_{n}$ and $\epsilon \in\{-1,1\}^{n}$. If $\epsilon_{i}=1$ then $\omega(i)$ does not have a bar over it and if $\epsilon(i)=-1$ then $\omega(i)$ has a bar over it. For example, the signed permutation $\overline{3} 54 \overline{2} 1$ can be expressed as $(35421,-111-11)$. A signed permutation $(\omega, \epsilon)$ maps $\left(x_{1}, \ldots, x_{n}\right) \in \mathbb{R}^{n}$ to $\left(\epsilon_{1} x_{\omega(1)}, \ldots, \epsilon_{n} x_{\omega(n)}\right)$.

The arrangement $\mathcal{B}_{n}$ is essential and has $2^{n} n$ ! regions which are all simplicial cones and are in a natural one-to-one correspondence with the elements of the hyperoctahedral group $B_{n}$. Under this correspondence a signed permutation $(\omega, \epsilon) \in B_{n}$ corresponds to the region

$$
R_{\omega, \epsilon}=\left\{\mathrm{x} \in \mathbb{R}^{n}: 0<\epsilon_{1} x_{\omega(1)}<\epsilon_{2} x_{\omega(2)}<\cdots<\epsilon_{n} x_{\omega(n)}\right\} .
$$

The intersection lattice $L_{\mathcal{B}_{n}}$ is isomorphic to the signed partition lattice $\Pi_{n}^{B}$ which is defined as follows. Let $\pi$ be a partition of the set $\{0,1, \ldots, n\}$. The block containing 0 is called the zero block. To bar an element of a block of $\pi$ is to place a bar above the element and to unbar a barred element is to remove the bar. A signed partition is a partition of the set $\{0,1, \ldots, n\}$ in which any of the nonminimal elements of any of the nonzero blocks are barred. For example,

$$
057|1 \overline{2} 9| 3 \overline{4} \overline{6} 8
$$

is a signed partition of $\{0,1, \ldots, 9\}$. It will be convenient to sometimes express a barred letter $\bar{a}$ of a signed partition as $(a,-1)$ and an unbarred letter as $(a, 1)$.

To bar a block $b$ in a signed partition is to bar all unbarred elements in $b$ and to unbar all barred elements in $b$. We denote this by $\bar{b}$. To unbar a block $b$ is to unbar all barred elements of $b$. We denote this by $\widetilde{b}$. For example,

$$
\overline{3 \overline{4} \overline{6} 8}=\overline{3} 46 \overline{8} \text { and } \widetilde{3 \overline{4} \overline{6} 8}=3468
$$

Let $\Pi_{n}^{B}$ be the poset of signed partitions of $\{0,1, \ldots, n\}$ with order relation defined by $\pi \leq \tau$ if for each block $b$ of $\pi$, either $b$ is contained in a nonzero block of $\tau, \bar{b}$ is contained in a nonzero block of $\tau$ or $\widetilde{b}$ is contained in the zero block of $\tau$. For example

$$
\begin{aligned}
& 057|1 \overline{2} 9| 3 \overline{4} \overline{6} 8<057 \mid 1 \overline{2} 93 \overline{4} \overline{6} 8 \\
& 057|1 \overline{2} 9| 3 \overline{4} \overline{6} 8<057 \mid 1 \overline{2} 9 \overline{3} 46 \overline{8}
\end{aligned}
$$

and

$$
057 \text { | } 1 \overline{2} 9 \text { | } 3 \overline{4} \overline{6} 8<0573468 \mid 1 \overline{2} 9 \text {. }
$$


The poset $\Pi_{n}^{B}$ is an example of a Dowling lattice [11].

For each signed partition $\pi \in \Pi_{n}^{B}$, let $\ell_{\pi}$ be the linear subspace of $\mathbb{R}^{n}$ consisting of all points $\left(x_{1}, x_{2}, \ldots, x_{n}\right)$ such that

- $x_{i}=x_{j}$ whenever $i$ and $j$ are in the same block of $\pi$ and both are barred or both are unbarred,

- $x_{i}=-x_{j}$ whenever $i$ and $j$ are in the same block of $\pi$ and one is barred and the other is unbarred,

- $x_{i}=0$ whenever $i$ is in the zero block.

The map $\pi \mapsto \ell_{\pi}$ is an isomorphism from $\Pi_{n}^{B}$ to $L_{\mathcal{B}_{n}}$. Let $\gamma$ be the inverse of this isomorphism.

The cycle $\rho_{R_{\omega, \epsilon}} \in \widetilde{H}_{n-2}\left(\bar{L}_{\mathcal{B}_{n}}\right)$ maps under $\gamma$ to the fundamental cycle of a spherical subposet of $\Pi_{n}^{B}$, which we now describe. To split a signed permutation $(\omega, \epsilon)$ at positions $i_{1}<\cdots<i_{k}$ in $\{0,1, \ldots, n-1\}$ is to form the signed partition with blocks $b_{0}, b_{1}, \ldots, b_{k}$ where $b_{0}=\left\{0, \omega(1), \ldots, \omega\left(i_{1}\right)\right\}$ (here $\left.\omega(0)=0\right)$, and for all $j=1, \ldots, k$,

$$
\left\{\left(\omega\left(i_{j}+1\right), \epsilon_{i_{j}+1}\right), \ldots,\left(\omega\left(i_{j+1}\right), \epsilon_{i_{j+1}}\right)\right\}
$$

is either $b_{j}$ or $\bar{b}_{j}$ (here $i_{k+1}=n$ ). For example, if we split the signed permutation

$$
\overline{3} 56 \overline{1} 87 \overline{4} 2
$$

at positions 2,5 we get the signed partition

$$
035|\overline{6} 1 \overline{8}| 7 \overline{4} 2 .
$$

For each signed permutation $(\omega, \epsilon) \in B_{n}$, let $\Pi_{\omega, \epsilon}$ be the induced subposet of $\Pi_{n}^{B}$ consisting of all signed partitions obtained by splitting the signed permutation $(\omega, \epsilon)$. Just as for type A, $\bar{\Pi}_{\omega, \epsilon}$ is spherical because $\Pi_{\omega, \epsilon}$ is isomorphic to the lattice of subsets of $[n]$.

Proposition 7.1. For all $(\omega, \epsilon) \in B_{n}$, the image $\gamma\left(\rho_{R_{\omega, \epsilon}}\right)$ is the fundamental cycle of $\bar{\Pi}_{\omega, \epsilon}$.

Proof. Let $z: P_{\mathcal{B}_{n}} \rightarrow L_{\mathcal{B}_{n}}$ be the map that takes cells to their affine span (cf. Section 3). We show that $\gamma$ restricts to an isomorphism from the subposet $z\left(P_{R_{\omega, \epsilon}}\right)$ of $L_{\mathcal{B}_{n}}$ to $\Pi_{\omega, \epsilon}$. Observe that elements of the face lattice $P_{R_{\omega, \epsilon}}$ are sets of the form

$$
\begin{array}{r}
\left\{\mathbf{x} \in \mathbb{R}^{n} \mid 0=x_{\omega(1)}=\cdots=x_{\omega\left(i_{1}\right)}<\epsilon_{i_{1}+1} x_{\omega\left(i_{1}+1\right)}=\cdots=\epsilon_{i_{2}} x_{\omega\left(i_{2}\right)}<\ldots\right. \\
\left.\cdots<\epsilon_{i_{k}+1} x_{\omega\left(i_{k}+1\right)}=\cdots=\epsilon_{n} x_{\omega(n)}\right\},
\end{array}
$$

where $0 \leq i_{1}<\cdots<i_{k} \leq n-1$. The linear span of such a set is the subspace

$$
\begin{array}{r}
\left\{\mathbf{x} \in \mathbb{R}^{n} \mid 0=x_{\omega(1)}=\cdots=x_{\omega\left(i_{1}\right)}, \epsilon_{i_{1}+1} x_{\omega\left(i_{1}+1\right)}=\cdots=\epsilon_{i_{2}} x_{\omega\left(i_{2}\right)}, \ldots\right. \\
\left.\ldots, \epsilon_{i_{k}+1} x_{\omega\left(i_{k}+1\right)}=\cdots=\epsilon_{n} x_{\omega(n)}\right\} .
\end{array}
$$


Hence $z\left(P_{R_{\omega, \epsilon}}\right)$ is the poset of subspaces of the form given in (4) ordered by reverse inclusion. Clearly $\gamma$ takes the subspace given in (4) to the signed partition obtained by splitting $(\omega, \epsilon)$ at positions $i_{1}, i_{2}, \ldots, i_{k}$.

Let $(\omega, \epsilon) \in B_{n}$. We say that $\omega(i)$ is a right-to-left maximum of $(\omega, \epsilon)$ if $\omega(i)>\omega(j)$ for all $j>i$.

Proposition 7.2. Let $\mathbf{v}=\left(1,2,2^{2}, \ldots, 2^{n-1}\right)$. Then the affine hyperplane $H_{\mathbf{v}}$ is generic with respect to the arrangement $\mathcal{B}_{n}$. Moreover, for all $(\omega, \epsilon) \in B_{n}, R_{\omega, \epsilon} \cap H_{\mathbf{v}}$ is bounded if and only if all right-to-left maxima of $(\omega, \epsilon)$ are unbarred.

Proof. The 1-dimensional intersections of hyperplanes in $\mathcal{B}_{n}$ have the form

$$
X=\left\{\mathbf{x} \in \mathbb{R}^{n} \mid 0=x_{i_{1}}=x_{i_{2}}=\cdots=x_{i_{k}}, \epsilon_{k+1} x_{i_{k+1}}=\cdots=\epsilon_{n} x_{i_{n}}\right\}
$$

where $0 \leq k \leq n-1,\left\{\left\{0, i_{1}, \ldots, i_{k}\right\},\left\{i_{k+1}, \ldots, i_{n}\right\}\right\}$ is a partition of $\{0,1, \ldots, n\}$ and $\epsilon_{i}= \pm 1$, for $i=k+1, \ldots, n$. Let $\mathbf{y} \in \mathbb{R}^{n}$ be defined by

$$
y_{i_{j}}= \begin{cases}0 & j=1, \ldots k \\ \epsilon_{j} & j=k+1, \ldots, n .\end{cases}
$$

One can easily check that

$$
\frac{4^{n}-1}{3 \sum_{t=k+1}^{n} \epsilon_{t} 2^{i_{t}-1}} \mathbf{y} \in H_{\mathbf{v}} \cap X .
$$

Hence $H_{\mathbf{v}} \cap X \neq \emptyset$ for all 1 -dimensional spaces $X$ in $L_{\mathcal{B}_{n}}$, which means that $H_{\mathbf{v}}$ is generic.

Now suppose $(\omega, \epsilon) \in B_{n}$ and $R_{\omega, \epsilon}$ is bounded. Assume that $\omega(m)$ is a right-to-left maximum that is barred. We will reach a contradiction of Lemma 4.3 by producing a vector $\mathbf{y}$ in the closure of $R_{\omega, \epsilon}$ such that $\mathbf{v} \cdot \mathbf{y}<0$. Let $\mathbf{y} \in \mathbb{R}^{n}$ be such that

$$
y_{\omega(i)}= \begin{cases}0 & \text { if } 1 \leq i<m \\ \epsilon_{i} & \text { if } m \leq i \leq n\end{cases}
$$

Clearly $\mathbf{y} \in \operatorname{cl}\left(R_{\omega, \epsilon}\right)$. We have

$$
\begin{aligned}
\mathbf{v} \cdot \mathbf{y} & =\sum_{j=m}^{n} 2^{\omega(j)-1} \epsilon_{j} \\
& \leq-2^{\omega(m)-1}+\sum_{j=m+1}^{n} 2^{\omega(j)-1} .
\end{aligned}
$$

Since $\omega(m)>\omega(j)$ for all $j>m$, we have

$$
\sum_{j=m+1}^{n} 2^{\omega(j)-1} \leq \sum_{i=1}^{\omega(m)-1} 2^{i-1}=2^{\omega(m)-1}-1 .
$$


Combining this with the previous inequality yields $\mathbf{v} \cdot \mathbf{y} \leq-1$.

Now suppose $(\omega, \epsilon) \in B_{n}$ and all right-to-left maxima of $(\omega, \epsilon)$ are unbarred. Let $m_{1}<m_{2}<\cdots<m_{k}$ be the positions of the right-to-left maxima. Let $\mathbf{x} \in R_{\omega, \epsilon}$. Then

$$
\begin{aligned}
\mathbf{v} \cdot \mathbf{x} & =\sum_{i=1}^{n} 2^{\omega(i)-1} x_{\omega(i)} \\
& =\sum_{j=1}^{k} \sum_{i=m_{j-1}+1}^{m_{j}} 2^{\omega(i)-1} x_{\omega(i)}
\end{aligned}
$$

where $m_{0}=0$. We have

$$
\omega(i)<\omega\left(m_{j}\right)
$$

for all $i=m_{j-1}+1, \ldots, m_{j}-1$, since $\omega\left(m_{j}\right)$ is a right-to-left maximum and $\omega(i)$ is not. Also,

$$
0<\epsilon_{i} x_{\omega(i)}<x_{\omega\left(m_{j}\right)}
$$

for all $i=m_{j-1}+1, \ldots, m_{j}-1$, since $\omega\left(m_{j}\right)$ is unbarred. Consequently

$$
\begin{aligned}
\sum_{i=m_{j-1}+1}^{m_{j}} 2^{\omega(i)-1} x_{\omega(i)} & \geq 2^{\omega\left(m_{j}\right)-1} x_{\omega\left(m_{j}\right)}-\sum_{\substack{i=m_{j-1}+1 \\
\epsilon_{i}<0}}^{m_{j}-1} 2^{\omega(i)-1} \epsilon_{i} x_{\omega(i)} \\
& \geq 2^{\omega\left(m_{j}\right)-1} x_{\omega\left(m_{j}\right)}-\sum_{\substack{i=m_{j-1}+1 \\
\epsilon_{i}<0}}^{m_{j}} 2^{\omega(i)-1} x_{\omega\left(m_{j}\right)} \\
& =x_{\omega\left(m_{j}\right)}\left(2^{\omega\left(m_{j}\right)-1}-\sum_{i=m_{j-1}+1}^{m_{j}-1} 2^{\omega(i)-1}\right) \\
& \geq x_{\omega\left(m_{j}\right)}\left(2^{\omega\left(m_{j}\right)-1}-\left(2^{\omega\left(m_{j}\right)-1}-1\right)\right) \\
& >0 .
\end{aligned}
$$

Thus we have shown that $\mathbf{v} \cdot \mathbf{x}>0$. By Lemma 4.3, $R_{\omega, \epsilon} \cap H_{\mathbf{v}}$ is bounded.

Theorem 7.3 (type B splitting basis). For $(\omega, \epsilon) \in B_{n}$, let $\rho_{\omega, \epsilon}$ be the fundamental cycle of the spherical poset $\bar{\Pi}_{\omega, \epsilon}$. Then

$$
\left\{\rho_{\omega, \epsilon} \mid(\omega, \epsilon) \in B_{n} \text { and all right-to-left maxima of }(\omega, \epsilon) \text { are unbarred }\right\}
$$

is a basis for $\widetilde{H}_{n-2}\left(\overline{\Pi_{n}^{B}}\right)$.

Proof. This follows from Theorem 4.2 and Propositions 7.1 and 7.2.

Corollary 7.4 (Dowling [11]). The rank of $\widetilde{H}_{n-2}\left(\overline{\Pi_{n}^{B}}\right)$ is

$$
1 \cdot 3 \cdot 5 \cdots(2 n-1) \text {. }
$$


Proof. One can construct a signed permutation by inserting (barred or unbarred) elements $n, n-1, \ldots, 1$ one at a time from largest to smallest. To construct a signed permutation in which the right-to-left maxima are unbarred, first we insert $n$ into the empty word. There is only one way to do this since $n$ must be unbarred. Now suppose we have inserted $n, n-1, \ldots, n-j+1$. If we insert $n-j$ at the end of the partially constructed word, $n-j$ will be a right-to-left maximum in the final word and must be unbarred. If we insert $n-j$ in any of the other $j$ positions $n-j$ will not be a right-to-left maximum and can therefore be barred or unbarred. Hence there are $2 j+1$ ways to insert $n-j$, for each $j=0,1, \ldots, n-1$.

Remark 7.5. The posets $\Pi_{n}$ and $\Pi_{n}^{B}$ are examples of Dowling lattices. Gottlieb and Wachs [13, Section 9] have constructed splitting bases for general Dowling lattices. The splitting basis for $\Pi_{n}$ is a special case of this Dowling lattice construction. For $\Pi_{n}^{B}$, the Dowling lattice construction produces the basis consisting of cycles $\rho_{\omega, \epsilon}$ where $(\omega, \epsilon) \in B_{n}$ is such that all left-to-right (rather than right-to-left) maxima of $(\omega, \epsilon)$ are unbarred. Although this basis is similar in appearance to the type $B$ splitting basis given here, one can show that it cannot be obtained from our geometric construction. (Consider the intersection of $\mathcal{B}_{3}$ with the cube as in Figure 4. The union of the closure of the regions corresponding to the signed permutations whose left-to-right maxima are unbarred, is not simply connected. Hence these regions cannot be the bounded regions of a generic slice.) However by using techniques similar to that of [13], one can get a variation of the Dowling lattice splitting basis which does reduce to the type $B$ splitting basis given here.

Although the only Dowling lattices that are intersection lattices of real hyperplane arrangements are the partition lattice and the signed partition lattice, there are other Dowling lattices that are intersection lattices of complex hyperplane arrangements (cf. [13, Section 8]). It would be interesting to find a geometric interpretation of the above mentioned variation of the splitting basis for such Dowling lattices.

Remark 7.6. The splitting basis of type $A$ is used in [20] and [21] to obtain information about the representation of the symmetric group on the homology of the partition lattice. Unfortunately, the splitting basis of type $B$ (or $D$ ) does not appear to reveal much about the representation of the Coxeter group on homology. See [15], [1] and [13] for work on the representation of $B_{n}$ on the homology of the signed partition lattice.

\section{Type $D$}

The type $D$ Coxeter arrangement is the hyperplane arrangement

$$
\mathcal{D}_{n}=\left\{x_{i}=x_{j} \mid 1 \leq i<j \leq n\right\} \cup\left\{x_{i}=-x_{j} \mid 1 \leq i<j \leq n\right\} .
$$

The orthogonal reflections across the hyperplanes generate the Coxeter group $D_{n}$, which is the subgroup of $B_{n}$ consisting of all signed permutations that have an even number of bars. Clearly $\mathcal{D}_{n}$ is a subarrangement of $\mathcal{B}_{n}$ and its intersection lattice is isomorphic to $\Pi_{n}^{D}$, the join-sublattice of $\Pi_{n}^{B}$ consisting of all signed partitions whose zero block does not 
have size 2. This isomorphism is denoted by $\gamma: L_{\mathcal{D}_{n}} \rightarrow \Pi_{n}^{D}$ and is the restriction of the isomorphism $\gamma: L_{\mathcal{B}_{n}} \rightarrow \Pi_{n}^{B}$ defined in the previous section.

The arrangement $\mathcal{D}_{n}$ is essential and has $2^{n-1} n$ ! regions which are simplicial cones in one-to-one correspondence with the elements of $D_{n}$. Under this correspondence the signed permutation $(\omega, \epsilon)$ corresponds to the region

$$
\widetilde{R}_{\omega, \epsilon}=\left\{\mathbf{x} \in \mathbb{R}^{n}|| x_{\omega(1)} \mid<\epsilon_{2} x_{\omega(2)}<\cdots<\epsilon_{n} x_{\omega(n)}\right\} .
$$

Note that the hyperplane $x_{w(1)}=0$ divides the region $\widetilde{R}_{\omega, \epsilon}$ into the regions $R_{\omega, \epsilon}$ and $R_{\omega, \epsilon^{\prime}}$, where $\epsilon_{i}^{\prime}=\epsilon_{i}$ for $i=2,3, \ldots, n$ and $\epsilon_{1}^{\prime}=-\epsilon_{1}$.

For each signed permutation $(\omega, \epsilon) \in D_{n}$, let $\widetilde{\Pi}_{\omega, \epsilon}$ be the induced subposet of $\Pi_{n}^{D}$ consisting of all signed partitions obtained by splitting either the signed permutation $(\omega, \epsilon)$ or the signed permutation $\left(\omega, \epsilon^{\prime}\right)$ at all positions in a subset of $\{0,1, \ldots, n-1\}$ whose smallest element is not 1 . Although it is not as evident as for types $A$ and $B$, this induced subposet is also a subset lattice.

Proposition 8.1. The induced subposet $\widetilde{\Pi}_{\omega, \epsilon}$ of $\Pi_{n}^{D}$ is isomorphic to the lattice of subsets of $[n]$.

Proof. Define the map $f: 2^{\{0,1, \ldots, n-1\}} \rightarrow \widetilde{\Pi}_{\omega, \epsilon}$ by letting $f(S)$ be the signed partition obtained by splitting $(\omega, \epsilon)$ at all positions in $S$ if 1 is not the smallest element of $S$, and by splitting $\left(\omega, \epsilon^{\prime}\right)$ at all positions in $S-\{1\} \cup\{0\}$ otherwise. We leave it to the reader to check that this map is an isomorphism from the dual of $2^{\{0,1, \ldots, n-1\}}$ to $\widetilde{\Pi}_{\omega, \epsilon}$.

Proposition 8.2. For all $(\omega, \epsilon) \in D_{n}$, the image $\gamma\left(\rho_{\widetilde{R}_{\omega, \epsilon}}\right)$ is the fundamental cycle of $\overline{\widetilde{\Pi}}_{\omega, \epsilon}$.

Proof. We show that $\gamma$ restricts to an isomorphism from the subposet $z\left(P_{\widetilde{R}_{\omega, \epsilon}}\right)$ of $L_{\mathcal{D}_{n}}$ to $\widetilde{\Pi}_{\omega, \epsilon}$. The elements of $P_{\widetilde{R}_{\omega, \epsilon}}$ are sets that have one of the following forms:

$$
\begin{aligned}
& \left\{\mathbf{x} \in \mathbb{R}^{n} \mid 0=x_{\omega(1)}=\cdots=x_{\omega\left(i_{1}\right)}<\epsilon_{i_{1}+1} x_{\omega\left(i_{1}+1\right)}=\cdots=\epsilon_{i_{2}} x_{\omega\left(i_{2}\right)}<\cdots\right. \\
& \left.\cdots<\epsilon_{i_{k}+1} x_{\omega\left(i_{k}+1\right)}=\cdots=\epsilon_{n} x_{\omega(n)}\right\} \text {, } \\
& \left\{\mathbf{x} \in \mathbb{R}^{n} \mid 0<x_{\omega(1)}=\epsilon_{2} x_{\omega(2)}=\cdots=\epsilon_{i_{1}} x_{\omega\left(i_{1}\right)}<\epsilon_{i_{1}+1} x_{\omega\left(i_{1}+1\right)}=\cdots=\epsilon_{i_{2}} x_{\omega\left(i_{2}\right)}\right. \\
& \left.<\cdots<\epsilon_{i_{k}+1} x_{\omega\left(i_{k}+1\right)}=\cdots=\epsilon_{n} x_{\omega(n)}\right\}, \\
& \left\{\mathbf{x} \in \mathbb{R}^{n} \mid 0<-x_{\omega(1)}=\epsilon_{2} x_{\omega(2)}=\cdots=\epsilon_{i_{1}} x_{\omega\left(i_{1}\right)}<\epsilon_{i_{1}+1} x_{\omega\left(i_{1}+1\right)}=\cdots=\epsilon_{i_{2}} x_{\omega\left(i_{2}\right)}\right. \\
& \left.<\cdots<\epsilon_{i_{k}+1} x_{\omega\left(i_{k}+1\right)}=\cdots=\epsilon_{n} x_{\omega(n)}\right\}, \\
& \left\{\mathbf{x} \in \mathbb{R}^{n}|0<| x_{\omega(1)} \mid<\epsilon_{2} x_{\omega(2)}=\cdots=\epsilon_{i_{1}} x_{\omega\left(i_{1}\right)}<\epsilon_{i_{1}+1} x_{\omega\left(i_{1}+1\right)}=\cdots=\epsilon_{i_{2}} x_{\omega\left(i_{2}\right)}\right. \\
& \left.<\cdots<\epsilon_{i_{k}+1} x_{\omega\left(i_{k}+1\right)}=\cdots=\epsilon_{n} x_{\omega(n)}\right\},
\end{aligned}
$$


where $2 \leq i_{1}<\cdots<i_{k}<n$.

The linear span of the cells are the respective subspaces:

$$
\begin{aligned}
& \left\{\mathbf{x} \in \mathbb{R}^{n} \mid 0=x_{\omega(1)}=\cdots=x_{\omega\left(i_{1}\right)}, \epsilon_{i_{1}+1} x_{\omega\left(i_{1}+1\right)}=\cdots=\epsilon_{i_{2}} x_{\omega\left(i_{2}\right)}, \ldots\right. \\
& \left.\ldots, \epsilon_{i_{k}+1} x_{\omega\left(i_{k}+1\right)}=\cdots=\epsilon_{n} x_{\omega(n)}\right\}, \\
& \left\{\mathbf{x} \in \mathbb{R}^{n} \mid x_{\omega(1)}=\epsilon_{2} x_{\omega(2)}=\cdots=\epsilon_{i_{1}} x_{\omega\left(i_{1}\right)}, \epsilon_{i_{1}+1} x_{\omega\left(i_{1}+1\right)}=\cdots=\epsilon_{i_{2}} x_{\omega\left(i_{2}\right)}, \ldots\right. \\
& \left.\ldots, \epsilon_{i_{k}+1} x_{\omega\left(i_{k}+1\right)}=\cdots=\epsilon_{n} x_{\omega(n)}\right\}, \\
& \left\{\mathbf{x} \in \mathbb{R}^{n} \mid-x_{\omega(1)}=\epsilon_{2} x_{\omega(2)}=\cdots=\epsilon_{i_{1}} x_{\omega\left(i_{1}\right)}, \epsilon_{i_{1}+1} x_{\omega\left(i_{1}+1\right)}=\cdots=\epsilon_{i_{2}} x_{\omega\left(i_{2}\right)}, \ldots\right. \\
& \left.\ldots, \epsilon_{i_{k}+1} x_{\omega\left(i_{k}+1\right)}=\cdots=\epsilon_{n} x_{\omega(n)}\right\} \\
& \left\{\mathbf{x} \in \mathbb{R}^{n} \mid \epsilon_{2} x_{\omega(2)}=\cdots=\epsilon_{i_{1}} x_{\omega\left(i_{1}\right)}, \epsilon_{i_{1}+1} x_{\omega\left(i_{1}+1\right)}=\cdots=\epsilon_{i_{2}} x_{\omega\left(i_{2}\right)}, \ldots\right. \\
& \left.\ldots, \epsilon_{i_{k}+1} x_{\omega\left(i_{k}+1\right)}=\cdots=\epsilon_{n} x_{\omega(n)}\right\},
\end{aligned}
$$

where $2 \leq i_{1}<\cdots<i_{k}<n$.

Clearly the images of such subspaces under $\gamma$ are precisely the elements of $\widetilde{\Pi}_{\omega, \epsilon}$.

Proposition 8.3. Let $\mathbf{v}=\left(1,2,2^{2}, \ldots, 2^{n-1}\right)$. Then the affine hyperplane $H_{\mathbf{v}}$ is generic with respect to the arrangement $\mathcal{D}_{n}$. Moreover, for all $(\omega, \epsilon) \in D_{n}, \widetilde{R}_{\omega, \epsilon} \cap H_{\mathbf{v}}$ is bounded if and only if $\omega(1) \neq n$ and all right-to-left maxima of $(\omega, \epsilon)$ are unbarred.

Proof. Since by Proposition $7.2, H_{\mathbf{v}}$ is generic with respect to the arrangement $\mathcal{B}_{n}$, it is generic with respect to any subarrangement of $\mathcal{B}_{n}$; in particular it is generic with respect to $\mathcal{D}_{n}$.

Since the hyperplane $x_{\omega(1)}=0$ divides $\widetilde{R}_{\omega, \epsilon}$ into the regions $R_{\omega, \epsilon}$ and $R_{\omega, \epsilon^{\prime}}$, the region $\widetilde{R}_{\omega, \epsilon} \cap H_{\mathbf{v}}$ is bounded if and only if both $R_{\omega, \epsilon} \cap H_{\mathbf{v}}$ and $R_{\omega, \epsilon^{\prime}} \cap H_{\mathbf{v}}$ are bounded. By Proposition 7.2, both regions are bounded if and only if all right-to-left maxima of both $(\omega, \epsilon)$ and $\left(\omega, \epsilon^{\prime}\right)$ are unbarred. This happens if and only if $\omega(1)$ is not a right-to-left maximum, i.e., $\omega(1) \neq n$, and all right-to-left maxima of $(\omega, \epsilon)$ are unbarred.

Theorem 8.4 (type D splitting basis). For each $(\omega, \epsilon) \in D_{n}$, let $\widetilde{\rho}_{\omega, \epsilon}$ be the fundamental cycle of $\bar{\Pi}_{\omega, \epsilon}$. Then

$$
\begin{gathered}
\left\{\widetilde{\rho}_{\omega, \epsilon} \mid(\omega, \epsilon) \in D_{n}, \omega(1) \neq n\right. \text { and all right-to-left maxima of } \\
(\omega, \epsilon) \text { are unbarred }\}
\end{gathered}
$$

is a basis for $\widetilde{H}_{n-2}\left(\overline{\Pi_{n}^{D}}\right)$.

Proof. This follows from Theorem 4.2 and Propositions 8.2 and 8.3.

The following corollary is well-known in the theory of Coxeter arrangements. 
Corollary 8.5. The rank of $\widetilde{H}_{n-2}\left(\overline{\Pi_{n}^{D}}\right)$ is

$$
1 \cdot 3 \cdot 5 \cdots(2 n-3) \cdot(n-1) .
$$

Proof. We construct a signed permutation $(\omega, \epsilon) \in D_{n}$ with all right-to-left maxima unbarred and $\omega(1) \neq n$ by first choosing $\omega(1)$ in $n-1$ ways and then choosing the signed permutation $\left(\omega(2) \omega(3) \ldots \omega(n), \epsilon_{2} \epsilon_{3} \cdots \epsilon_{n}\right)$ so that every right-to-left maximum is unbarred. By the proof of Corollary 7.4, this signed permutation on $n-1$ letters can be chosen in $1 \cdot 3 \cdots(2 n-3)$ ways. If the number of bars in $\left(\omega(2) \omega(3) \ldots \omega(n), \epsilon_{2} \epsilon_{3} \cdots \epsilon_{n}\right)$ is even then $\omega(1)$ must be unbarred; otherwise $\omega(1)$ must be barred.

\section{$9 \quad$ Interpolating partition lattices}

We now consider a family of posets which interpolates between the type $\mathrm{D}$ partition lattice and the type B partition lattice. For $T \subseteq[n]$, let $\Pi_{n}^{D B}(T)$ be the join-sublattice of $\Pi_{n}^{B}$ consisting of all signed partitions whose zero block is not $\{0, a\}$ for $a \in[n] \backslash T$. Clearly $\Pi_{n}^{D B}(\emptyset)=\Pi_{n}^{D}$ and $\Pi_{n}^{D B}([n])=\Pi_{n}^{B}$. The lattice $\Pi_{n}^{D B}(T)$ is the intersection lattice of the hyperplane arrangement

$$
\begin{aligned}
\mathcal{D B}_{n}(T)= & \left\{x_{i}=x_{j} \mid 1 \leq i<j \leq n\right\} \cup\left\{x_{i}=-x_{j} \mid 1 \leq i<j \leq n\right\} \\
& \cup\left\{x_{i}=0 \mid i \in T\right\} .
\end{aligned}
$$

These interpolating arrangements were introduced by Zaslavsky [24]. The following theorem generalizes Theorems 7.3 and 8.4.

Theorem 9.1. For each $(\omega, \epsilon) \in B_{n}$, let $\rho_{\omega, \epsilon}$ be the fundamental cycle of $\bar{\Pi}_{\omega, \epsilon}$, and if $(\omega, \epsilon) \in D_{n}$ let $\widetilde{\rho}_{\omega, \epsilon}$ be the fundamental cycle of $\widetilde{\widetilde{\Pi}}_{\omega, \epsilon}$. The set

$$
\begin{gathered}
\left\{\rho_{\omega, \epsilon} \mid(\omega, \epsilon) \in B_{n}, \omega(1) \in T\right. \text { and all right-to-left maxima of } \\
(\omega, \epsilon) \text { are unbarred }\} \\
\cup \\
\left\{\widetilde{\rho}_{\omega, \epsilon} \mid(\omega, \epsilon) \in D_{n}, \omega(1) \notin T \cup\{n\}\right. \text { and all right-to-left maxima of } \\
(\omega, \epsilon) \text { are unbarred }\}
\end{gathered}
$$

forms a basis for $\widetilde{H}_{n-2}\left(\overline{\Pi_{n}^{D B}(T)}\right)$.

Proof. There are two types of regions of the hyperplane arrangement $\mathcal{D} \mathcal{B}_{n}(T)$, namely $R_{\omega, \epsilon}$ for $(\omega, \epsilon) \in B_{n}$ and $\omega(1) \in T$, and $\widetilde{R}_{\omega, \epsilon}$ for $(\omega, \epsilon) \in D_{n}$ and $\omega(1) \notin T$. By Propositions 7.1 and 8.2, $\rho_{\omega, \epsilon}$ and $\widetilde{\rho}_{\omega, \epsilon}$ are the respective images (up to sign) of $\rho_{R_{\omega, \epsilon}}$ and $\rho_{\widetilde{R}_{\omega, \epsilon}}$, under the isomorphism $\gamma$.

We proceed as in the proof of Theorem 8.4. For $\mathbf{v}=\left(1,2, \ldots, 2^{n-1}\right), H_{\mathbf{v}}$ is generic with respect to the arrangement $\mathcal{D} \mathcal{B}_{n}(T)$. Hence the result now follows from Theorem 4.2, Propositions 7.2 and 8.3. 
Corollary 9.2 (Jambu and Terao [16]). The rank of $\widetilde{H}_{n-2}\left(\overline{\Pi_{n}^{D B}(T)}\right)$ is

$$
1 \cdot 3 \cdot 5 \cdots(2 n-3) \cdot(|T|+n-1) .
$$

Proof. Suppose $n \in T$. Then the number of signed permutations $(\omega, \epsilon) \in B_{n}$ such that $\omega(1) \in T$ and all right-to-left maxima are unbarred is $(2|T|-1) \cdot 1 \cdot 3 \cdots(2 n-3)$. The number of signed permutations $(\omega, \epsilon) \in D_{n}$ such that $\omega(1) \notin T \cup\{n\}$ and all right-to-left maxima are unbarred is $(n-|T|) \cdot 1 \cdot 3 \cdots(2 n-3)$.

Now suppose $n \notin T$. Then the number of signed permutations $(\omega, \epsilon) \in B_{n}$ such that $\omega(1) \in T$ and all right-to-left maxima are unbarred is $2|T| \cdot 1 \cdot 3 \cdots(2 n-3)$. The number of signed permutations $(\omega, \epsilon) \in D_{n}$ such that $\omega(1) \notin T \cup\{n\}$ and all right-to-left maxima are unbarred is $(n-|T|-1) \cdot 1 \cdot 3 \cdots(2 n-3)$. In either case, the total number of elements in the basis is $(|T|+n-1) \cdot 1 \cdot 3 \cdots(2 n-3)$.

Józefiak and Sagan [17] have studied other families of hyperplane arrangements which interpolate between Coxeter arrangements. One can apply our results to these arrangements. The family of arrangements that interpolate between $\mathcal{A}_{n-2}$ and $\mathcal{A}_{n-1}$ are particularly amenable to our approach. For $T \subseteq[n-1]$, let $\mathcal{A}_{n}(T)$ be the arrangement in $\mathbb{R}^{n}$,

$$
\mathcal{A}_{n}(T)=\left\{x_{i}=x_{j} \mid 1 \leq i<j \leq n-1\right\} \cup\left\{x_{n}=x_{i} \mid i \in T\right\} .
$$

Let $\Pi_{n}(T)$ be the induced subposet of $\Pi_{n}$ consisting of all partitions $\pi$ such that the block of $\pi$ containing $n$ is either a singleton or has nonempty intersection with $T$. This is the intersection lattice of $\mathcal{A}_{n}(T)$. The following theorem generalizes Theorem 6.3 by providing a splitting basis for the homology of $\Pi_{n}(T)$. Recall that in Section 6 we defined $\rho_{\omega}$ to be the fundamental cycle of $\bar{\Pi}_{\omega}$, for each $\omega \in S_{n}$.

Theorem 9.3. For $\emptyset \neq T \subseteq[n-1]$, the set

$$
\left\{\rho_{\omega} \mid \omega \in S_{n}, \omega(n)=n \text { and } \omega(n-1) \in T\right\}
$$

forms a basis for $\widetilde{H}_{n-3}\left(\overline{\Pi_{n}(T)}\right)$.

Proof. The proof, which is similar to that of Theorem 9.1, uses results from Section 6 and is left to the reader.

Remark 9.4. There are easier and more direct ways to prove Theorem 9.3. For instance, one can restrict the second EL-labeling for $\Pi_{n}$ given in the proof of Theorem 6.2 of [20], to $\Pi_{n}(T)$. The induced shelling basis is precisely the basis given in Theorem 9.3.

Corollary 9.5 (Józefiak and Sagan [17]). For $\emptyset \neq T \subseteq[n-1]$, the rank of $\widetilde{H}_{n-3}\left(\overline{\Pi_{n}(T)}\right)$ is $(n-2) ! \cdot|T|$.

In [19], Stanley showed that the restriction to $S_{n-1}$ of the representation of $S_{n}$ on $\widetilde{H}_{n-3}\left(\Pi_{n}, \mathbb{C}\right)$ is the regular representation. In [20], it was observed that the splitting basis for $\widetilde{H}_{n-3}\left(\overline{\Pi_{n}}\right)$ makes this fact transparent. Indeed, the permutations that fix $n$ permute the basis cycles $\rho_{\omega}$. A similar phenomenon occurs for $\Pi_{n}(T)$. 
Corollary 9.6. For $\emptyset \neq T \subseteq[n-1]$, the representation of $S_{T} \times S_{[n-1] \backslash T}$ on $\widetilde{H}_{n-3}\left(\overline{\Pi_{n}(T)}, \mathbb{C}\right)$ is isomorphic to the direct sum of $\left(\begin{array}{c}n-2 \\ |T|-1\end{array}\right)$ copies of the regular representation of $S_{T} \times$ $S_{[n-1] \backslash T}$.

Proof. If $\omega \in S_{n}$ satisfies $\omega(n)=n$ and $\omega(n-1) \in T$ then so does $\sigma \omega$ for all $\sigma \in$ $S_{T} \times S_{[n-1] \backslash T} \times S_{\{n\}}$. In fact, the elements of $T$ occupy the same set of positions in $\sigma \omega$ as in $\omega$. Since $\sigma \rho_{\omega}=\rho_{\sigma \omega}$, we see that $S_{T} \times S_{[n-1] \backslash T}$ acts on $\widetilde{H}_{n-3}\left(\overline{\bar{\Pi}_{n}(T)}, \mathbb{C}\right)$ by permuting basis cycles. Also, the orbit of $\rho_{\omega}$ is determined by the set of positions that elements of $T$ occupy in $\omega$. Hence the number of orbits is $\left(\begin{array}{c}n-2 \\ |T|-1\end{array}\right)$.

Remark 9.7. John Shareshian [personal communication] has found an alternative proof of Corollary 9.6 which involves computing the Möbius function of the $\sigma$-invariant subposet of $\Pi_{n}(T)$ for $\sigma \in S_{T} \times S_{[n-1] \backslash T}$ by means of Crapo's complementation formula [9].

Remark 9.8. Another class of partition posets with a splitting basis is the class of $d$ divisible partition lattices [20], or the more general restricted block size partition lattices considered in [7] and [8]. These are not geometric lattices in general; but they are intersection lattices of subspace arrangements and they are shellable. The cycles in the basis are polytopal. The symmetric group acts on these lattices and the splitting basis reveals much information about the representation of the symmetric group on homology. It would be interesting to find a geometric explanation for this spitting basis. One might also consider restricted block size partition subposets of $\Pi_{n}(T)$.

\section{References}

[1] N. Bergeron, A hyperoctahedral group analogue of the free Lie algebra, J. Combin. Theory A 58 (1991), 256-178.

[2] A. Björner, Shellable and Cohen-Macaulay partially ordered sets, Trans. Amer. Math. Soc. 260 (1980), 159-183.

[3] A. Björner, On the homology of geometric lattices, Algebra Universalis 14 (1982), $107-128$.

[4] A. Björner, The homology and shellability of matroids and geometric lattices, in "Matroid Applications" (ed. N. White), Cambridge Univ. Press, 1992, pp. 226-283.

[5] A. Björner, M. LasVergnas, B. Sturmfels, N. White and G. M. Ziegler, Oriented Matroids, Cambridge Univ. Press, 1993.

[6] A. Björner and M. L. Wachs, Shellable nonpure complexes and posets I, Trans. Amer. Math. Soc. 348 (1996), 1299-1327.

[7] A. E. Browdy, The (Co)Homology of Lattices of Partitions with Restricted Block Size, Ph.D. dissertation, University of Miami, 1996. 
[8] A. E Browdy and M. L. Wachs, The (co)homology of lattices of partitions with restricted block size, in preparation.

[9] H. H. Crapo, The Möbius function of a lattice, J. Combin. Theory 1 (1966), 126-131.

[10] J. Damon, Higher multiplicities and almost free divisors and complete intersections, Memoirs Amer. Math. Soc. 589, 1996.

[11] T. A. Dowling, A class of geometric lattices based on finite groups, J. Combin. Theory $B 14$ (1973), 61-86.

[12] J. Folkman, The homology groups of a lattice, J. Math. Mech. 15 (1966), 631-636.

[13] E. Gottlieb and M. L. Wachs, Cohomology of Dowling lattices and Lie (super) algebras, Advances in Applied Math. 24 (2000), 301-336.

[14] P. Hanlon, The fixed point partition lattices, Pacific J. Math. 96 (1981), 319-341.

[15] P. Hanlon, The characters of the wreath product group acting on the homology groups of the Dowling lattices, J. Algebra 91 (1984), 430-463.

[16] M. Jambu and H. Terao, Free arrangements of hyperplanes and supersolvable latties, Advances in Math. 52 (1984), 248-258.

[17] T. Józefiak and B. E. Sagan, Basic derivations for subarrangements of Coxeter arrangements, J. Algebraic Combin. 2 (1993), 291-320.

[18] D. Quillen, Homotopy properties of the poset of non-trivial p-subgroups of a group, Advances in Math. 28 (1978), 101-128.

[19] R. P. Stanley, Some aspects of groups acting on finite posets, J. Combin Theory A 32 (1982), 132-161.

[20] M. L. Wachs, A basis for the homology of the $d$-divisible partition lattice, Advances in Math. 117 (1996), 294-318.

[21] M. L. Wachs, On the (co)homology of the partition lattice and the free Lie algebra, Discrete Math. 193 (1998), 287-319.

[22] M. L. Wachs and J. W. Walker, On geometric semilattices, Order 2 (1986), 367-385.

[23] T. Zaslavsky, Facing up to arrangements: Face-count formulas for partitions of space by hyperplanes, Memoirs Amer. Math. Soc. 154, 1975.

[24] T. Zaslavsky, The geometry of root systems and signed graphs, Amer. Math. Monthly 88 (1981), 88-105.

[25] G. M. Ziegler, The face lattice of hyperplane arrangements, Discrete Math. 73 (1988), $233-238$.

[26] G. M. Ziegler, Matroid shellability, $\beta$-systems, and affine hyperplane arrangements, J. Algebraic Combin. 1 (1992), 283-300. 